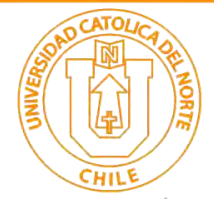

\title{
Edge even graceful labeling of torus grid graph
}

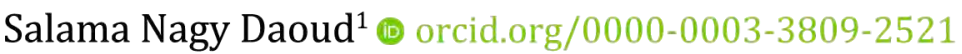

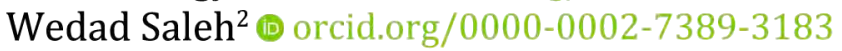

${ }^{1}$ Menoufia University, Dept. of Mathematics, Shebin El Kom, Egypt. Taibah University, Dept.of Mathematics, Al- Medina, Saudi Arabia.

- salamadaoud@gmail.com

${ }^{2}$ Taibah University, Dept.of Mathematics, Al- Medina, Saudi Arabia.

=wed_10_777@hotmail.com

\section{Abstract:}

We study the family of torus grid graphs. We also obtain necessary and sufficent conditions to be edge even graceful labeling for all of the cases of every member of this family.

Keywords: Graceful labeling; Edge even graceful labeling; Torus grid graph.

$\operatorname{MSC}(2020): 05 C 78$.

\section{Cite this article as (IEEE citation style):}

S. N. Daoud and W. Saleh, "Edge even graceful labeling of torus grid graph", Proyecciones (Antofagasta, On line), vol. 39, no. 4, pp. 1033-1082, Aug. 2020, doi: 10.22199/issn.0717-6279-202004-0065.

Article copyright: (C) 2020 Salama Nagy Daoud and Wedad Saleh. This is an open access article distributed under the terms of the Creative Commons License, which permits unrestricted use and distribution provided the original author and source are credited. 


\section{Introduction}

The scope of Graph Theory plays a very significant base in many areas of applied and pure sciences. One amidst the necessary areas in Graph Theory is Graph Labeling of a graph that is associate of integers either to the vertices or edges or each subject to assured conditions. Graph labeling began nearly fifty years ago. Over these decades, over two hundred ways of labeling techniques were given and over 2500 papers were revealed. In spite of this vastliterature, simply a number of general results were discovered. Today, graph labeling has a lot of attention from completely different good researches in Graph Theory that has rigorous applications in several disciplines such as communication networks, writing theory, graph decomposition problems and so on. Additional, fascinating applications of graph labeling are given in $[1,3,4,5,6,18,19,26,27,29]$.

A function $g$ is named a graceful labeling of a graph $G$ with vertices $n$ and edges $m$ if $g: V(G) \longrightarrow\{0,1,2, \cdots, m\}$ is $(1-1)$ and $g^{*}$ : $E(G) \longrightarrow\{1,2, \cdots, m\}$ is given as $g^{*}(e=u v)=|g(u)-g(v)|$ is correspondence. This kind of graph labeling first is obtained in [24] as a $\beta$ valuation, lately is called graceful labeling in [17]. A function $g$ is called an odd graceful labeling of a graph $G$ with vertices $n$ and edges $m$ if $g: v(G) \longrightarrow\{0,1,2, \cdots, 2 m-1\}$ is injective and the induced function $g^{*}: v(G) \longrightarrow\{1,3, \cdots, 2 m-1\}$ is defined as $g^{*}(e=u v)=|g(u)-g(v)|$ is one-to-one correspondence and we will use o.g.l referring to this type of labeling. Also, the readers can see $[15,16,25]$ for a lot of results. A function $g$ is named an edge graceful labeling of a graph $G$ with vertices $n$ and edges $m$ if $g: E(G) \longrightarrow\{1,2, \cdots, m\}$ is one-to-one correspondence and $g^{*}: V(G) \longrightarrow\{0,1,2, \cdots, n-1\}$ that is known as $g^{*}(u)=$ $\sum_{e-u v \in E(G)} g(e)(\bmod n)$ is one-to-one correspondence and we will use e.g.l referring to this type of labeling. Also, the readers can see $[20,21,23]$ for a lot of results. A function $g$ is named an edge odd graceful labeling of a graph $G$ with vertices $n$ and edges $m$ if $g: E(G) \longrightarrow\{1,3, \cdots, 2 m-1\}$ is one-to-one correspondence and $g^{*}: V(G) \longrightarrow\{0,1,2, \cdots, 2 m-1\}$ is given as $g^{*}(u)=\sum_{e-u v \in E(G)} g(e)(\bmod 2 m)$ is injective, will use e.o.g.l referring to this type of labeling. This type of graph labeling can be found ininitial introduced by Solairaju and Chithra in 2009 [28]. For more information on this labeling see $[7,8,10]$. A function $g$ is named an edge even graceful labeling of a graph $G$ with vertices $n$ and edges $m$ if $g: E(G) \longrightarrow\{2,4, \cdots, 2 m-2\}$ is one-to-one correspondence and 
$g^{*}: V(G) \longrightarrow\{0,2,4, \cdots, 2 m-2\}$ given as $g^{*}(u)=\sum_{e-u v \in E(G)} g(e)(\bmod 2 r)$, where $r=\max \{n, m\}$ is (1-1), we will use e.e.g.l referring to this type of labeling. This type of graph labeling can be found initial introduced by Elsonbaty and Daoud [11]. For more information on this labling see [9] and [14]. For more information about other types of graph labelings, see $[2,12,13,22,30]$

\section{Labeling of Torus grid Graph}

$T_{p, q}$ which is torus grid graph is a graph constructed from the Cartesian product $C_{p} \times C_{q}$ where $C_{p}$ and $C_{q}$ are the cycle graphs. That is the torus grid graph consists of $p$ copies of $C_{q}$ represented by circles and can be numbered from the inner most circle to the outer circle as: $C_{q}^{(1)}, C_{q}^{(2)}, C_{q}^{(3)}, \cdots, C_{q}^{(p-1)}, C_{q}^{(p)}$ and we decision them circles, also $q$ copies of $C_{p}$ represented by ovals or elliptic shapes transverse the $p$ circles and can be numbered clockwise as $C_{p}^{(1)}, C_{p}^{(2)}, C_{p}^{(3)}, \cdots, C_{p}^{(q-1)}, C_{p}^{(q)}$ and that we call them ovals. See Figure 2.1 . 


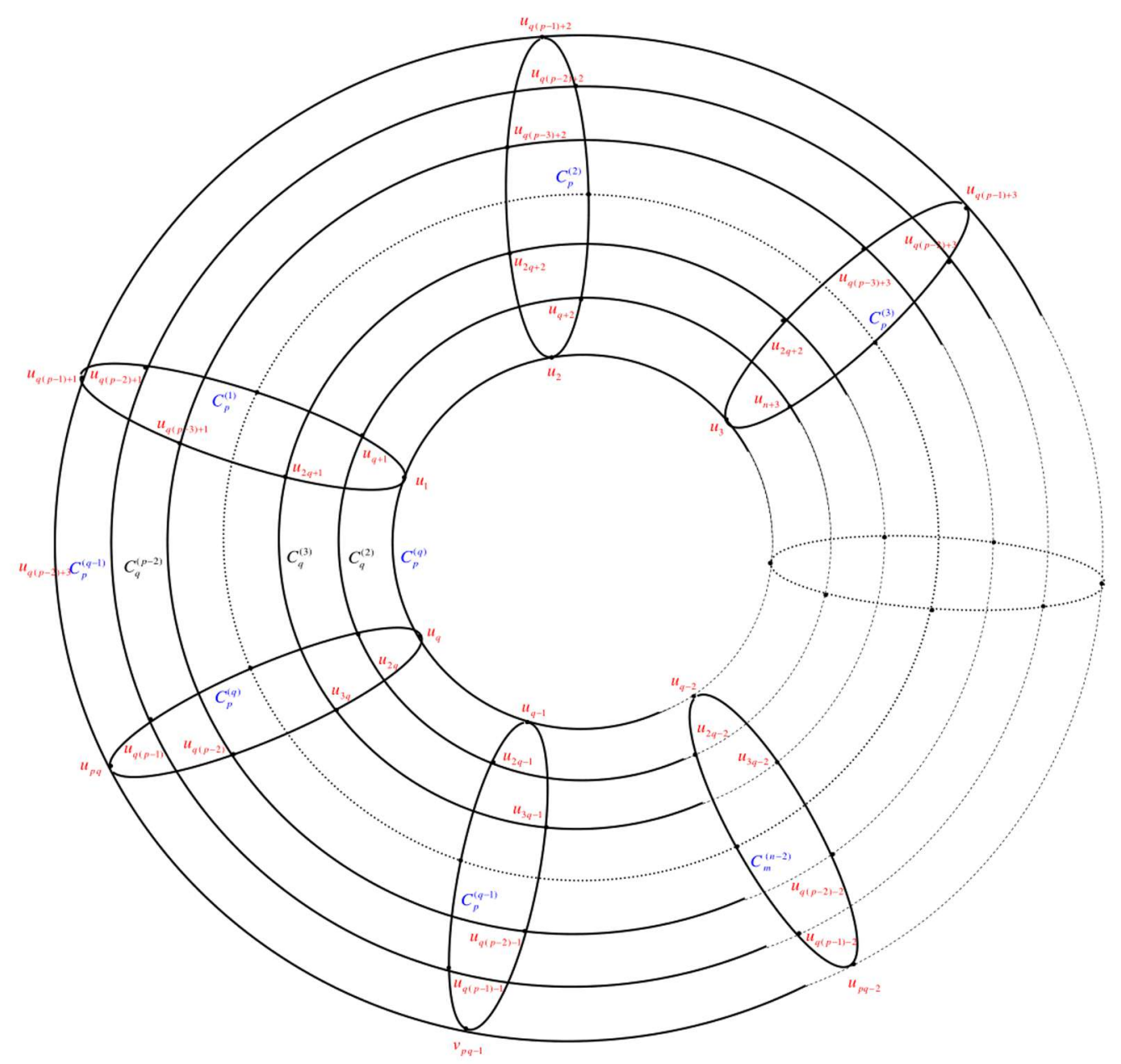

Figure 2.1: Tous grid graph $T_{p, q}$

Theorem 2.0.1. Let $q$ be an even number and it belongs to $\mathbf{Z}^{+}$and $2 \leq p$ is greater than or equal 2. Then $T_{p, q}$, is an e. e. g. graph. 
Proof. By taking $n=\left|V\left(T_{p, q}\right)\right|=p q, m=\left|E\left(T_{p, q}\right)\right|=2 p q$ and $r=$ $\max m, n=2 p q$ and $g: E\left(T_{p, q}\right) \longrightarrow\{2,4, \cdots, 2 p q-2\}$. Assume that $T_{p, q}$ is the torus grid graphis that is given in Figure 2.2. First we label the ovals $C_{p}^{(j)}, 1 \leq j \leq q$ as follows: turn clockwise to label the edges

$$
\begin{aligned}
& u_{(p-1) q+1} u_{(p-2) q+1}, u_{(p-1) q+2} u_{(p-2) q+2}, \\
& u_{(p-1) q+3} u_{(p-2) q+3}, \cdots, u_{p q-1} u_{(p-1) q-1}, u_{p q} u_{(p-1) q}
\end{aligned}
$$

by $2,4,6, \cdots 2 q-2,2 q$, then turn counter-clockwise to label the edges

$$
\begin{aligned}
& u_{(p-2) q+1} u_{(p-4) q+1}, u_{(p-1) q} u_{(p-3) q}, u_{(p-1) q-1} u_{(p-3) q-1}, \\
& \cdots, u_{(p-2) q+3} u_{(p-4) q+3}, u_{(p-2) q+2} u_{(p-4) q+2}
\end{aligned}
$$

by $2 q+2,2 q+4,2 q+6, \cdots, 4 q-2,4 q$ and so on.

When $p \equiv 0 \bmod 4$, we turn counter-clockwise to label the edges

$$
u_{2 q+1} u_{1}, u_{q} u_{3 q}, u_{q-1} u_{3 q-1}, \cdots, u_{2 q+3} u_{3}, u_{2 q+2} u_{2}
$$

by $(p-2) q+2,(p-2) q+4,(p-2) q+6, \cdots, p q-2, p q$, we turn clockwise to label the edges

$$
u_{1} u_{q+1}, u_{2} u_{q+2}, u_{3} u_{q+3}, \cdots, u_{q-1} u_{2 q-1}, u_{q} u_{2 q}
$$

by $p q+2, p q+4, p q+6, \cdots,(p+2) q-2,(p+2) q$, then turn counter-clockwise to label the edges

$$
u_{q+1} u_{3 q+1}, u_{2 q} u_{4 q}, u_{2 q-1} u_{4 q-1}, \cdots, u_{q+3} u_{3 q+3}, u_{q+2} u_{3 q+2}
$$

by $(p+2) q+2,(p+2) q,(p+2) q+6, \cdots,(p+4) q-2,(p+4) q$ and so on. When $p \equiv 2 \bmod 4$, turn clockwise to label the edges

$$
u_{2 q+1} u_{1}, u_{2 q+1} u_{2}, u_{2 q+3} u_{3}, \cdots, u_{3 q-1} u_{q-1}, u_{3 q} v_{q}
$$


by $(p-2) q+2,(p-2) q+4,(p-2) q+6, \cdots, p q-2, p q$, then turn counterclockwise anticlockwise to label the edges

$$
u_{1} u_{q+1}, u_{q} u_{2 q}, u_{q-1} u_{2 q-1}, \cdots, u_{3} u_{q+3}, u_{2} u_{q+2}
$$

by $p q+2, p q+4, p q+6, \cdots,(p+2) q-2,(p+2) n$, then turn clockwise to label the edges

$$
u_{q+1} u_{3 q+1}, u_{q+2} u_{3 q+2}, u_{q+3} u_{3 q+3}, \cdots, u_{2 q-1} u_{4 q-1}, u_{2 q} u_{4 q}
$$

by $(p+2) q+2,(p+2) q+4,(p+2) q+6, \cdots,(p+4) q-2,(p+4) q$ and so on.

Now, turn counter-clockwise to label the edges

$$
\begin{aligned}
& u_{(p-1) q-1} u_{(p-3) q-1}, u_{p q} u_{(p-2) q}, u_{p q-1} u_{(p-2) q-1}, \\
& \cdots, u_{(p-1) q+3} u_{(p-3) q+3}, u_{(p-1) q+2} u_{(p-3) q+2}
\end{aligned}
$$

by $2(p-1) q+2,2(p-1) q, 2(p-1) q+6, \cdots, 2 p q-2,2 p q$. 


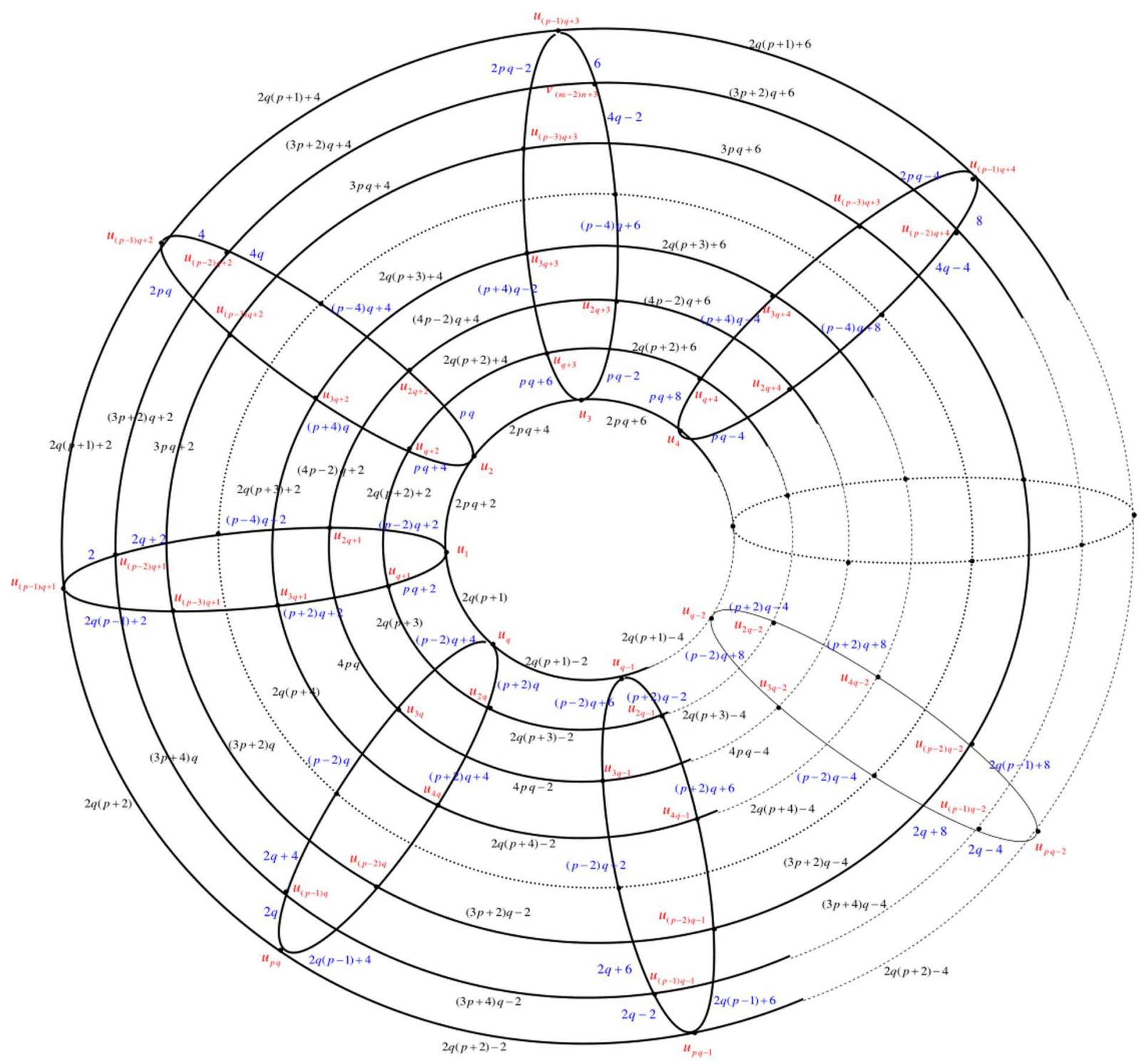

Figure 2.2: $T_{p, q} p \equiv 0 \bmod 4$

Second we label the edges of the circles $C_{q}^{(j)}, 1 \leq j \leq p$ begin with the edges of inner most circle $C_{q}^{(1)}$ then the edges of outer circle $C_{q}^{(p)}$, then the edges of the circles $C_{q}^{(2)}, C_{q}^{(4)}, \cdots, C_{q}^{(p-2)}$. 
Finally the edges of the circles $C_{q}^{(p-1)}, C_{q}^{(p-3)}, \cdots, C_{q}^{(3)}$, respectively as follows: $g\left(u_{i} u_{i+1}\right)=2 p q+2 i, 1 \leq i \leq q-1$,

$g\left(u_{q} u_{1}\right)=2(p+1) q, g\left(u_{(p-1) q+i} g_{(p-1) q+i+1}\right)=2 q(p+1)+2 i, 1 \leq i \leq q-1$,

$g\left(u_{p q} u_{(p-1) q+1}\right)=2 q(p+2), g\left(u_{(j-1) q+i} u_{(j-1) q+i+1}\right)=2 q\left(p+\frac{j+2}{2}\right)+2 i$,

$g\left(u_{j q} u_{(j-1) q+1}\right)=2 q\left(p+\frac{j+4}{2}\right), 2 \leq j \leq p-2, j$ is an even and $1 \leq i \leq q-1$, $g\left(u_{(j-1) q+i} u_{(j-1) q+i+1}\right)=(4 p-j+1) q+2 i, g\left(u_{j q} u_{(j-1) q+i+1}\right)=(4 p-(j-$ 3)) $q, 3 \leq j \leq p-1, k$ is odd and $1 \leq i \leq q-1$.

Thus the labels of corresponding vertices $\bmod 4 p q$ in case of $p \equiv 0 \bmod 4$ become :

$g^{*}\left(u_{i}\right) \equiv 2 p q+4 i+2, g^{*}\left(u_{2 q+i}\right) \equiv 2 p q-8 q+4 i+2, g^{*}\left(u_{4 q+i}\right) \equiv 2 p q-16 q+$ $4 i+2, \cdots g^{*}\left(u_{\left(\frac{p}{2}+3\right) q+i}\right) \equiv 20 q+4 i+2, \cdots, g^{*}\left(u_{(p-5) q+i}\right)=2 p q-12 q+$ $4 i+2, g^{*}\left(u_{(p-3) q+i}\right)=2 p q-4 q+4 i+2, g^{*}\left(u_{(p-1) q+i}\right)=2 p q+4 q+4 i+$ $2, g^{*}\left(u_{n+i}\right)=2 p n+12 n+4 i+2, g^{*}\left(u_{3 q+i}\right)=2 p q+20 q+4 i+2, g^{*}\left(u_{5 q+i}\right)=$ $2 p q+28 q+4 i+2, \cdots, g^{*}\left(u_{\left(\frac{p}{2}-7\right) q+i}\right) \equiv 4 p q-12 q+4 i+2, g^{*}\left(u_{\left(\frac{p}{2}-3\right) q+i}\right) \equiv$ $4 p q-4 q+4 i+2, \cdots, g^{*}\left(u_{\left(\frac{p}{2}\right) q+i}\right) \equiv 2 p q+8 q+4 i+2, g^{*}\left(u_{(p-4) q+i}\right) \equiv$ $2 p q+16 q+4 i+2$ and $g^{*}\left(u_{\left(\frac{p}{2}+2\right) q+i}\right) \equiv 4 p q+8 q+4 i+2,1 \leq i \leq q$. be:

In case of $p \equiv 2 \bmod 4$, the labels of corresponding vertices $\bmod 4 p q$ can $g^{*}\left(u_{i}\right) \equiv 2 p q+4 i+2, g^{*}\left(u_{2 q+i}\right) \equiv 2 p q-8 q+4 i+2, g^{*}\left(u_{4 q+i}\right) \equiv 2 p q-$ $16 q+4 i+2, \cdots, g^{*}\left(u_{\frac{p}{2}-5} q+i\right) \equiv 20 q+4 i+2, g^{*}\left(u_{\frac{p}{2}-3} q+i\right) \equiv 12 q+4 i+$ $2, g^{*}\left(u_{\frac{p}{2}-1} q+i\right) \equiv 4 p q-24 q+4 i+2, g^{*}\left(u_{\frac{p}{2}-6} q+i\right) \equiv 4 p q-16 q+4 i+$ $2, g^{*}\left(u_{\frac{p}{2}-4} q+i\right) \equiv 4 p q-8 q+4 i+2, g^{*}\left(u_{\frac{p}{2}-2} q+i\right) \equiv 4 i+2, g^{*}\left(u_{\frac{p q}{2}}+i\right) \equiv$ $8 q+4 i+2, g^{*}\left(u_{\frac{p}{2}+2}+i\right) \equiv 16 q+4 i+2, \cdots, g^{*}\left(u_{(p-5) q+i}\right) \equiv 2 p q-12 q+$ $4 i-2, g^{*}\left(u_{(p-3) q+i}\right) \equiv 2 p q-4 q+4 i-2, g^{*}\left(u_{(p-1) q+i}\right) \equiv 2 p q-20 q+4 i-$ $2, g^{*}\left(u_{\left(\frac{p}{2}+1\right) q+i}\right) \equiv 4 p q-4 q+4 i-2, g^{*}\left(u_{\left(\frac{p}{2}+3\right) q+i}\right) \equiv 4 p q-12 q+4 i-$ $2, g^{*}\left(u_{\left(\frac{p}{2}+5\right) q+i}\right) \equiv 4 p q-20 q+4 i-2, \cdots, g^{*}\left(u_{(p-6) q+i}\right) \equiv 2 p q+24 q+4 i+$ $2, g^{*}\left(u_{(p-4) q+i}\right) \equiv 2 p q+16 q+4 i+2, g^{*}\left(u_{(p-2) q+i}\right) \equiv 2 p q+8 q+4 i+22$, $1 \leq i \leq q$. 
Illustration: The e. e. g. 1. $T_{8,11}, T_{8,12}, T_{10,8}$ and $T_{10,9}$, respectively are displayed in Figure 2.3.

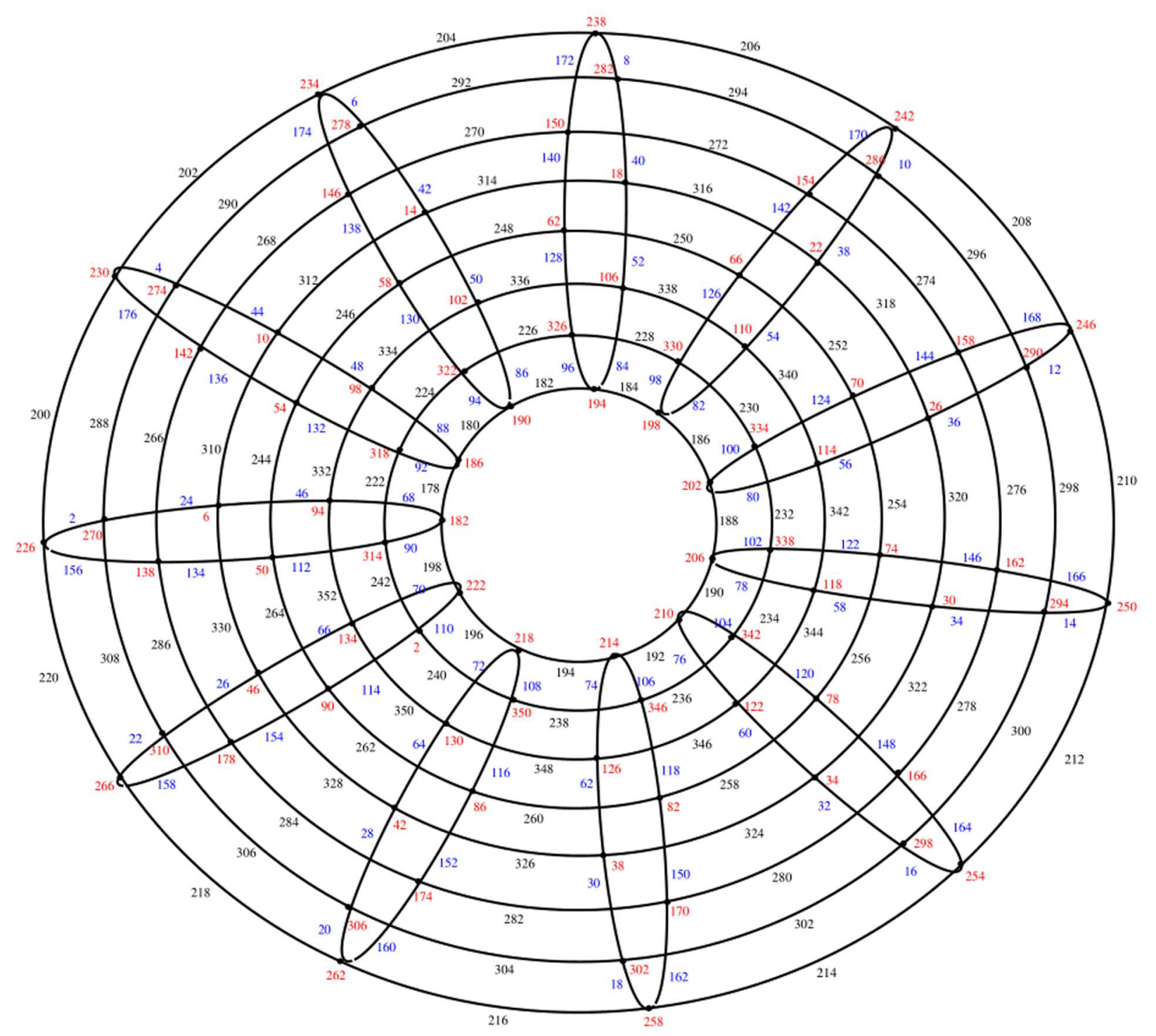

(a) $T_{8,11}$ 


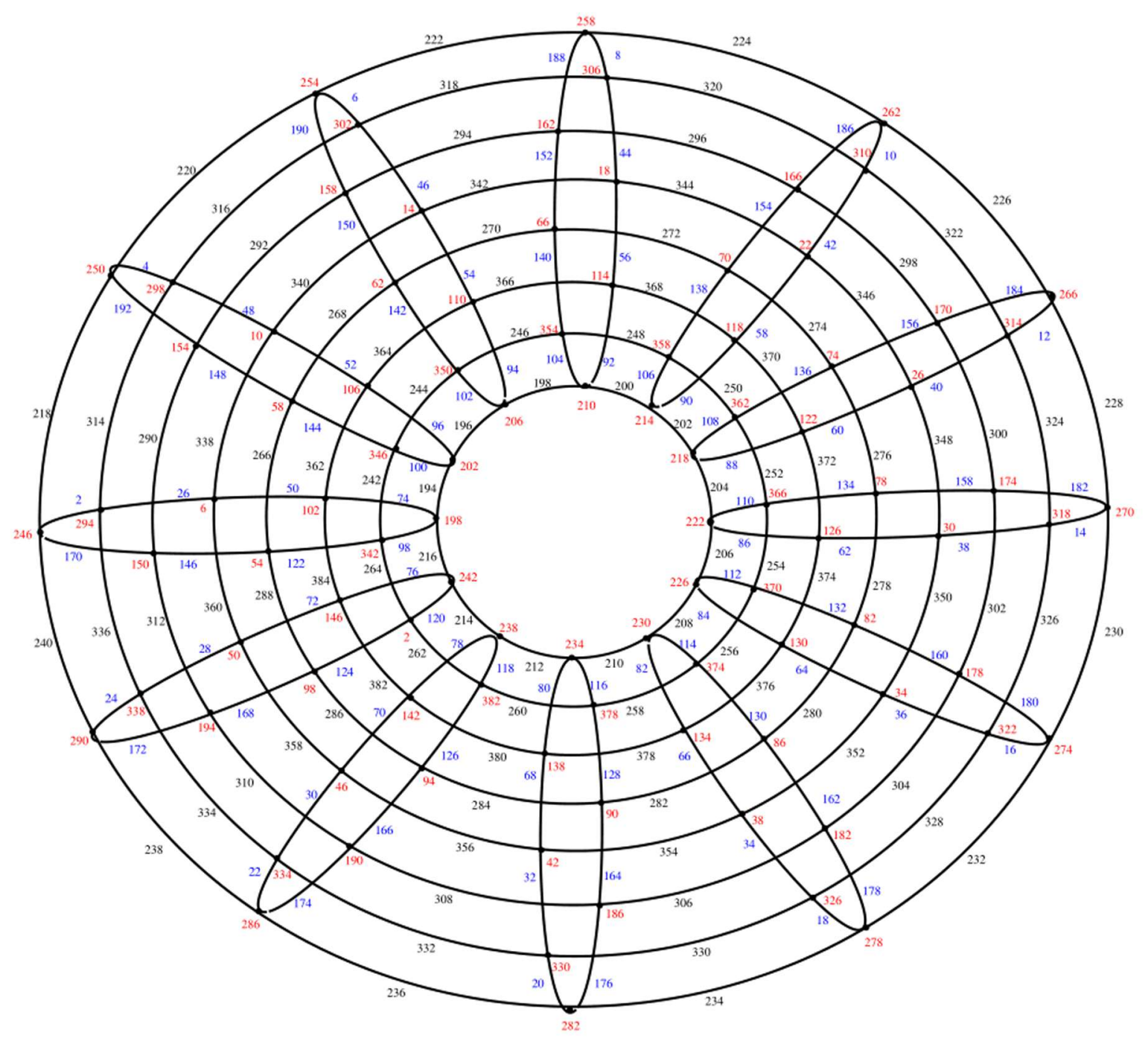

(b) $T_{8,12}$ 


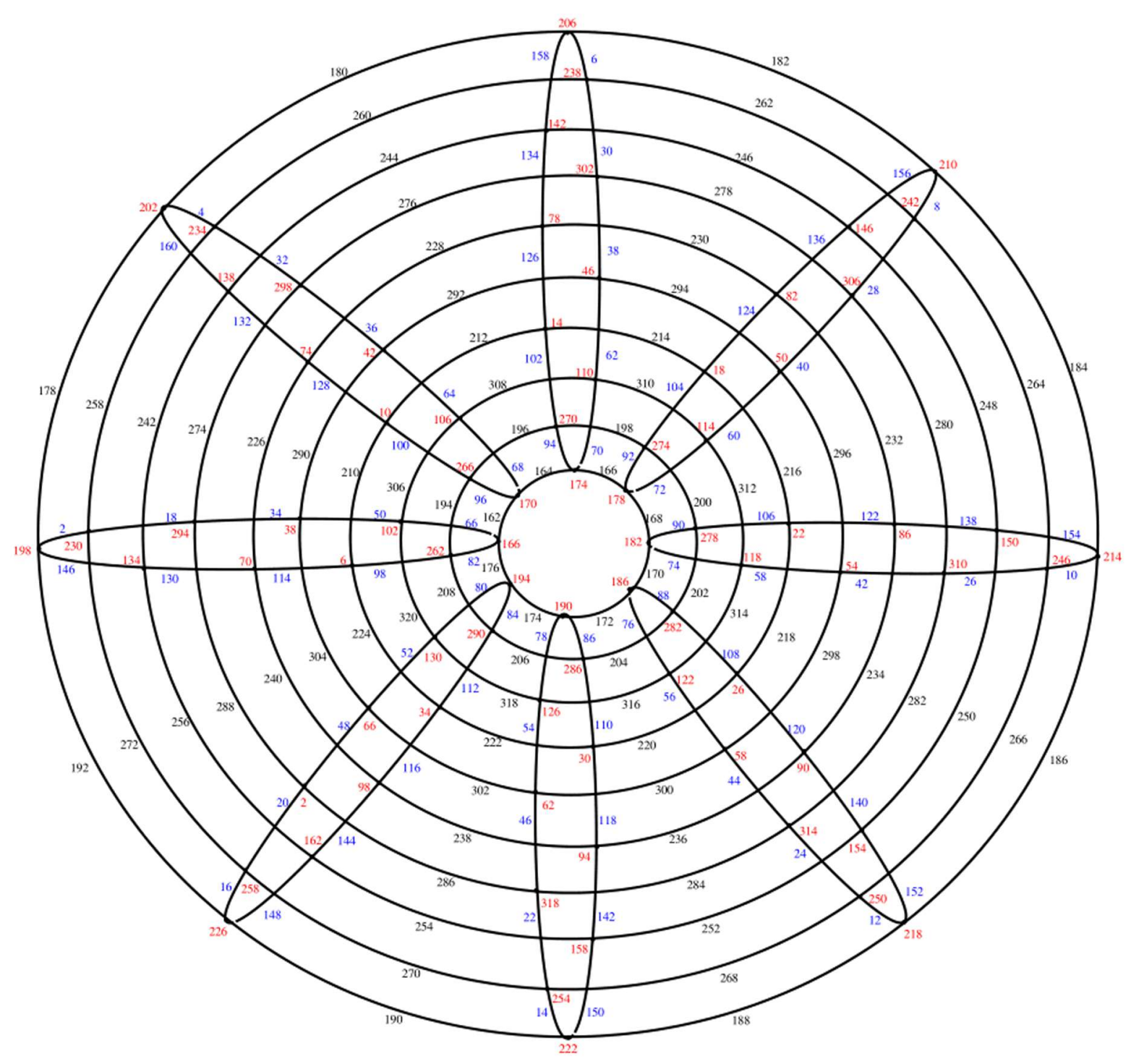

(c) $T_{10,8}$ 


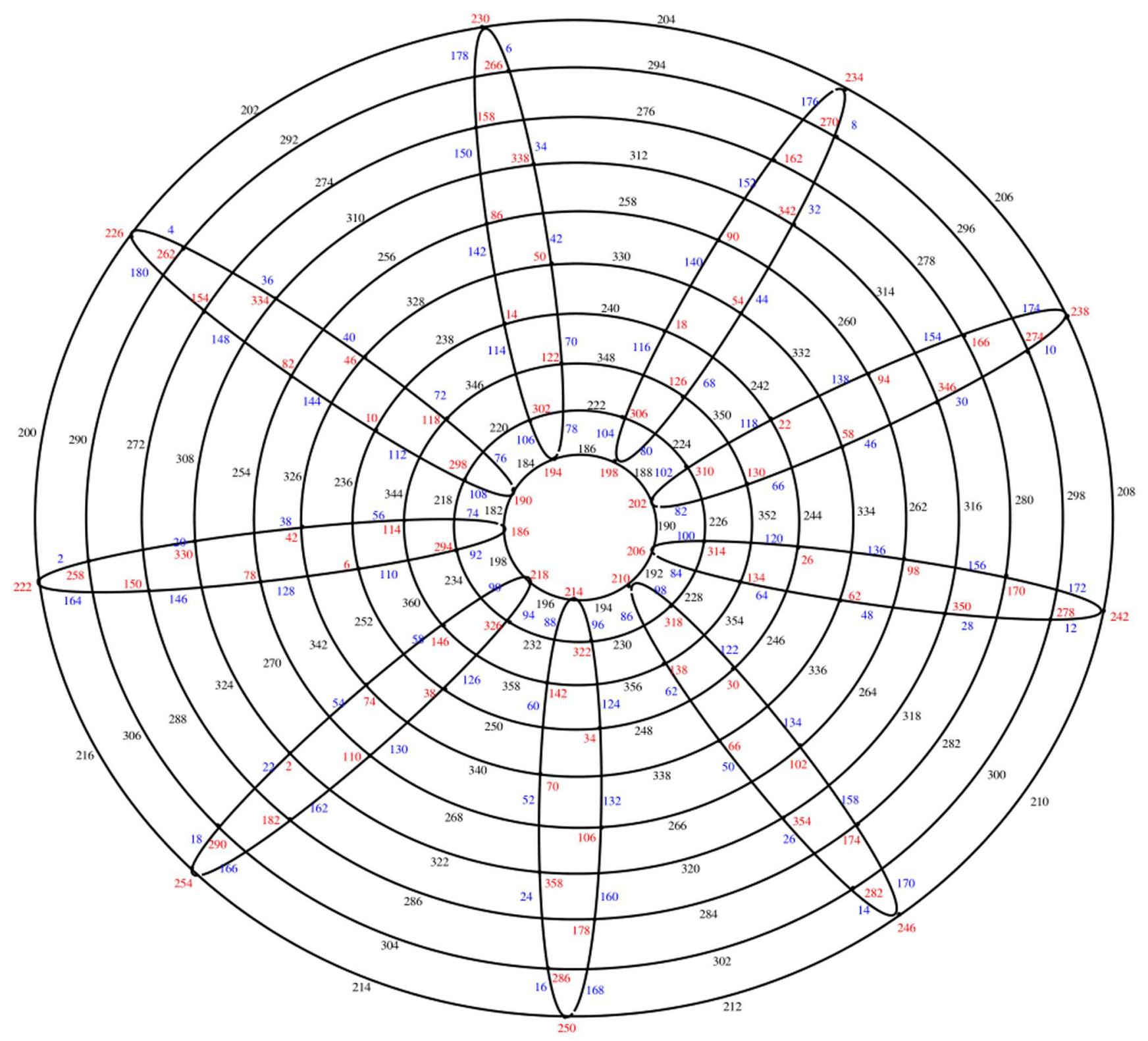

(d) $T_{10,9}$

Figure 2.3: The e. e. g. l. of $T_{8,11}, T_{8,12}, T_{10,8}$ and $T_{10,9}$ 
Lemma 2.0.2. If $q=2$ and $p>1$ is an odd number and belong to $\mathbf{Z}^{+}$. Then $T_{p, 2}$ is an e. e. g. graph.

Proof. Consider that $T_{p, 2}$ which is in Figure 2.4.

Now, let us label the edges of the left oval as follows:

$g\left(u_{2 p-1} u_{2 p-3}\right)=2 ; g\left(u_{2 p-(4 j-1)} u_{2 p-(4 j+3)}\right)=4 j+4,1 \leq j \leq \frac{p-3}{2}, g\left(u_{1} u_{3}\right)=$ $2 p+2 ; g\left(u_{4 j-3} u_{4 j+1}\right)=2 p+4 j+2,1 \leq j \leq \frac{p-1}{2}$.

Hence, label the edges of the right oval as follows:

$g\left(u_{4 j-2} u_{4 j+2}\right)=2 p+4 j+4 ; g\left(u_{2 p-4 j+2} u_{2 p-4 j-2}\right)=4 j+6,1 \leq j \leq$ $\frac{p-3}{2}, g\left(u_{2 p-4} u_{2 q}\right)=4 ; g\left(u_{2 p} u_{2 q-2}\right)=6, g\left(u_{2} u_{4}\right)=2 p+4$.

Moreover, we label the edges of the upper half circles as follows:

$g\left(u_{1} u_{2}\right)=4 p+2 ; g\left(u_{2 p-1} u_{2 p}\right) \equiv 4 j+6 ; g\left(u_{4 j-1} u_{4 j}\right)=4 p+8 j+2 ; g\left(u_{2 p-4 j-1} u_{2 p-4 j}\right)=$ $4 p+8 j+6,1 \leq j \leq \frac{p-3}{2}$ and $g\left(u_{2 p-3} u_{2 p-2}\right)=8 p-2$.

Finally, we label the edges of the lower half circles as follows:

$g\left(u_{1} u_{2}\right)=4 p+4 ; g\left(u_{2 p-1} u_{2 p}\right)=4 j+8 ; g\left(u_{4 j-1} u_{4 j}\right)=4 p+8 j+4 ; g\left(u_{2 p-4 j-1} u_{2 p-4 j}\right)=$ $4 p+8 j+8,1 \leq j \leq \frac{p-3}{2}$ and $g\left(u_{2 p-3} u_{2 p-2}\right)=8 m$.

Thus, the labels of the corresponding vertices $\bmod 8 p$ become: $g^{*}\left(u_{4 j+1}\right) \equiv 4 p-8 j+14,0 \leq j \leq \frac{p-3}{2} ; g^{*}\left(u_{4 j+2}\right) \equiv 4 p-8 j+18 ; g^{*}\left(u_{4 j+3}\right) \equiv$ $4 p-8 j+22 ; g^{*}\left(u_{4 j+4}\right) \equiv 4 p-8 j+26,1 \leq j \leq \frac{p-5}{2} ; g^{*}\left(u_{2 p-3}\right) \equiv 8 ; g^{*}\left(u_{2 p-2}\right) \equiv$ $14 ; g^{*}\left(u_{2 p-1}\right) \equiv 4 q+16 ; g^{*}\left(u_{2 p}\right) \equiv 24$ 


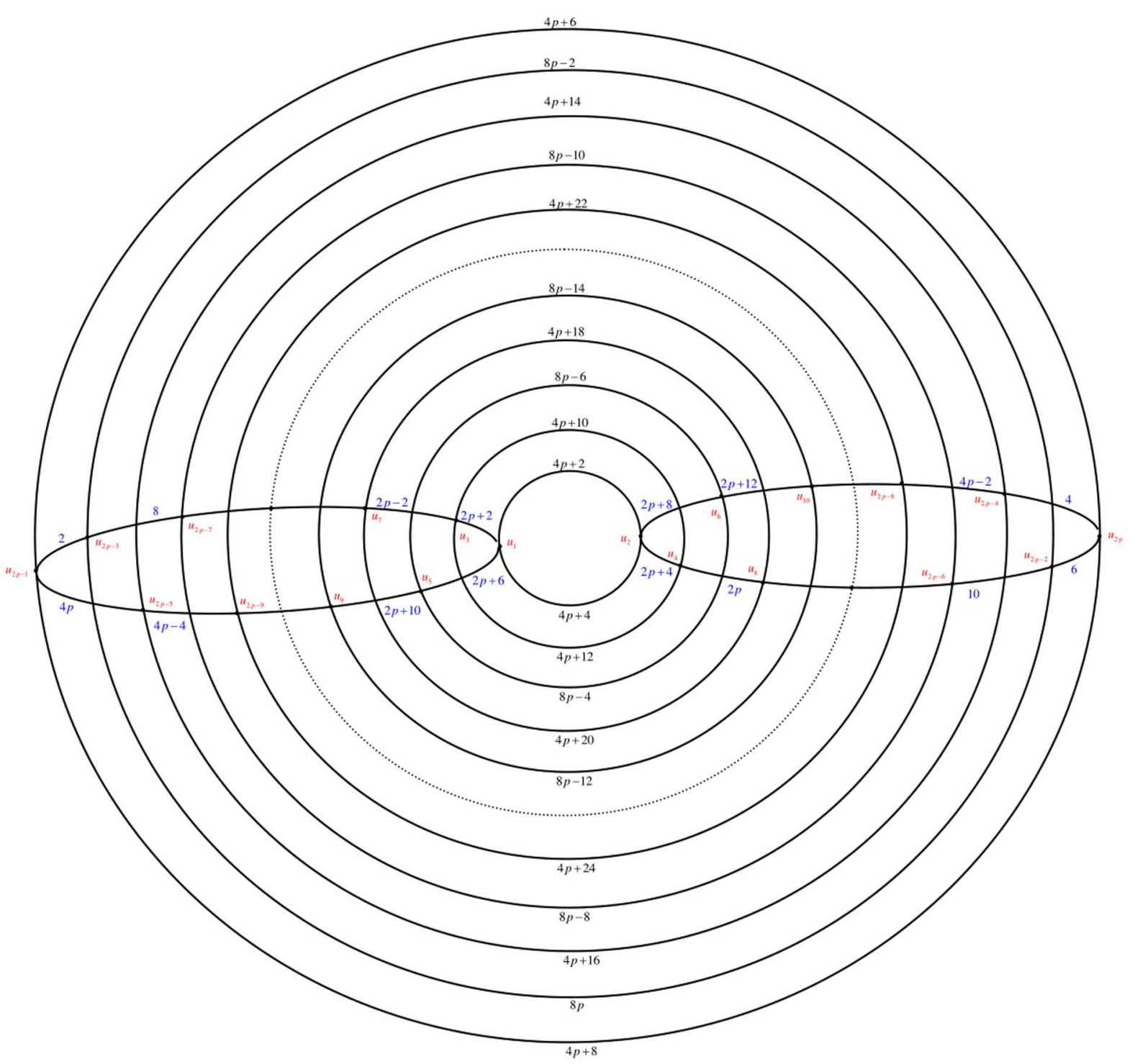

Figure 2.4: $T_{p, 2} p>1$ where $p$ is odd 
Illustration: The e. e. g. 1. of $T_{11,2}$ is presented in Figure 2.5.

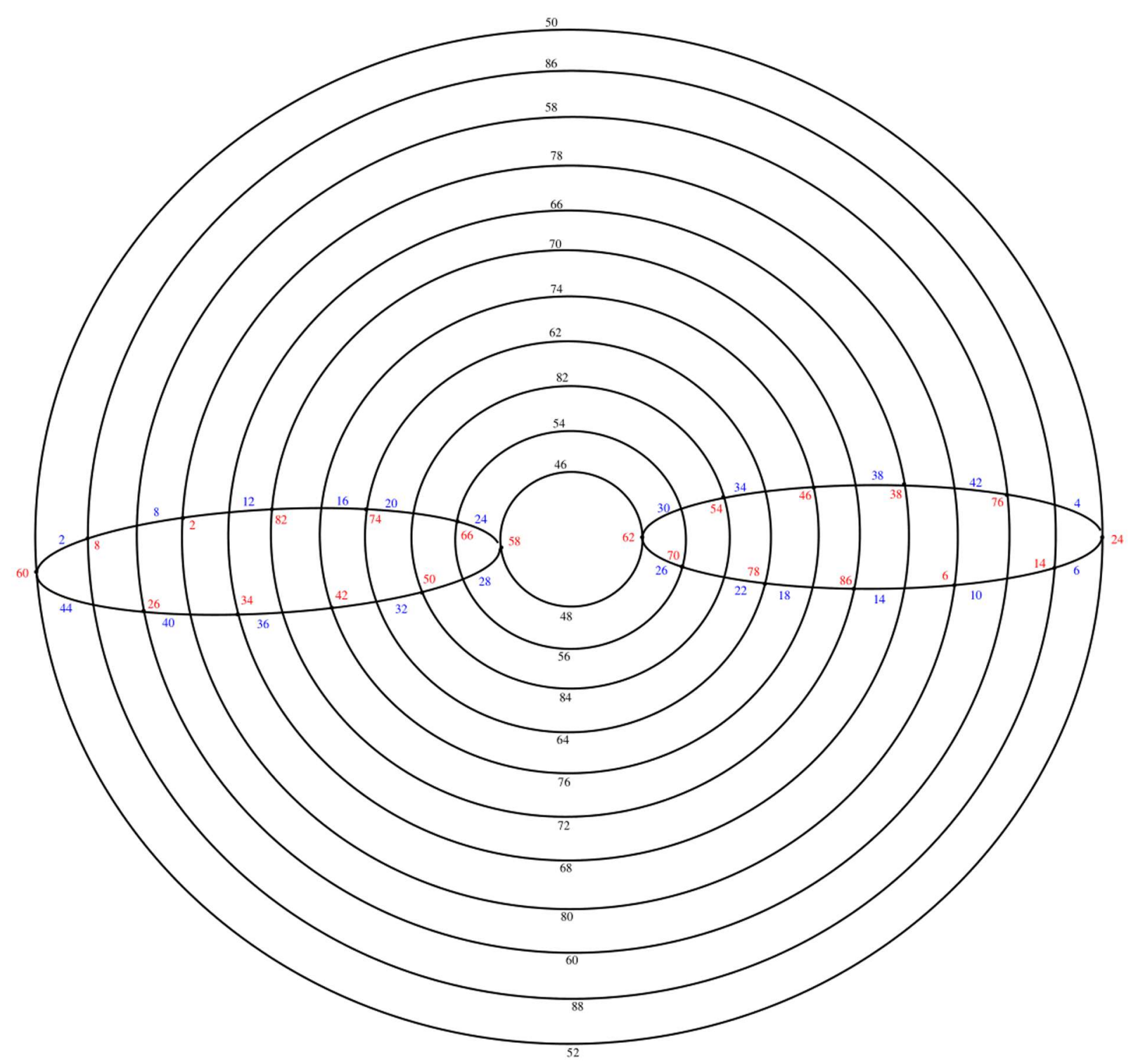

Figure 2.5: e. e. g. l. of $T_{11,2}$ 
Lemma 2.0.3. If $q=3$ and $p$ is an odd positive integer greater than 1 . Then the torus grid graph $T_{p, 3}$ is an edge even graceful graph.

Proof. Assume that $T_{p, 3}$ which is in Figure 2.6. We label the edges of the ovals begin with the edges of the oval $C_{p}^{(1)}$ to the edges of the oval $C_{p}^{(3)}$ such as: First, turn clockwise to label the edges $u_{3 m-2} u_{3 m-5}, u_{3 m-1} u_{3 m-4}, u_{3 m} u_{3 m-3}$ by $4,6,8$, then turn counter-clockwise to label the edges $u_{3 m-5} u_{3 m-11}, u_{3 m-3} u_{3 m-9}, u_{3 m-4} u_{3 m-11}$ by $10,12,14$ and so on.

Next, turn clockwise to label the edges $u_{4} u_{1}, u_{5} u_{2}, u_{6} u_{3}$ by $3 m+1,3 m+$ $3,3 m+5$, then turn counter-clockwise anticlockwise to label the edges $u_{1} u_{7}, u_{3} u_{9}, u_{2} u_{8}$ by $3 p+7,3 p+9,3 p+11$, then turn clockwise to label the edges $u_{7} u_{13}, u_{8} u_{14}, u_{9} u_{15}$ by $3 p+13,3 p+15,3 p+17$ and so on.

Now, turn clockwise to label the edges $u_{3 p-8} u_{3 p-2}, u_{3 p-7} u_{3 p-1}, u_{3 p} u_{3 p-3}$ by $6 p-2,6 p, 2$.

In this step, we label the edges of the circles $C_{3}^{(j)}, 1 \leq j \leq p$ begin with the edges of the inner most circle $C_{3}^{(1)}$, then the edges of the circle $C_{3}^{(p)}$ then the edges of the circles

$$
C_{3}^{(2)}, C_{3}^{(p-2)}, C_{3}^{(4)}, C_{3}^{(p-4)}, C_{3}^{(6)}, C_{3}^{(p-6)}, \cdots, C_{3}^{(3)}, C_{3}^{(p-1)}
$$

as follows:

$g\left(u_{1} u_{2}\right)=6 p+2 ; g\left(u_{2} u_{3}\right)=6 p+6 ; g\left(u_{3} u_{1}\right)=6 p+4 ; g\left(u_{3 p-2} u_{3 p-1}\right)=$ $6 p+8, g\left(u_{3 p-1} u_{3 p}\right)=6 p+12, g\left(u_{3 p} u_{3 p-2}\right)=6 p+10, g\left(u_{3 j-2} u_{3 j-1}\right)=6 p+$ $6 j+2, g\left(u_{3 j-1} u_{3 j}\right)=6 p+8 j+4, g\left(u_{3 j} u_{3 j-2}\right)=6 p+6 j+6,2 \leq j \leq p-1$ where $j$ is even and

$g\left(u_{3 j-2} u_{3 j-1}\right)=12 p-6 j+8, g\left(u_{3 j-1} u_{3 j}\right)=12 p-6 j+10, g\left(u_{3 j} u_{3 j-2}\right)=$ $12 p-6 j+12,3 \leq j \leq p-2$, where $j$ is odd.

Thus, the labels of the corresponding vertices $\bmod 12 m$ become:

$g^{*}\left(u_{1}\right) \equiv 6 p+14, g^{*}\left(u_{2}\right) \equiv 6 p+12, g^{*}\left(u_{3}\right) \equiv 6 p+24, g^{*}\left(u_{3 p-2}\right) \equiv 6 p+$ $20, g^{*}\left(u_{3 p-1}\right) \equiv 6 p+26, g^{*}\left(u_{3 p}\right) \equiv 32, g^{*}\left(u_{3 j+i-3}\right) \equiv 6 p+6 j+4 i+12,2 \leq i \leq$ $3,2 \leq j \leq p-1$ where $j$ is even; $g^{*}\left(u_{3 j+i-3}\right) \equiv 6 p-6 j+4 i+18,1 \leq i \leq 3,3 \leq$ $j \leq p-4$ where $j$ is odd; $g^{*}\left(u_{3 p-8}\right) \equiv 34, g^{*}\left(u_{3 p-7}\right) \equiv 38, g^{*}\left(u_{3 p}\right) \equiv 6 p+42$ 


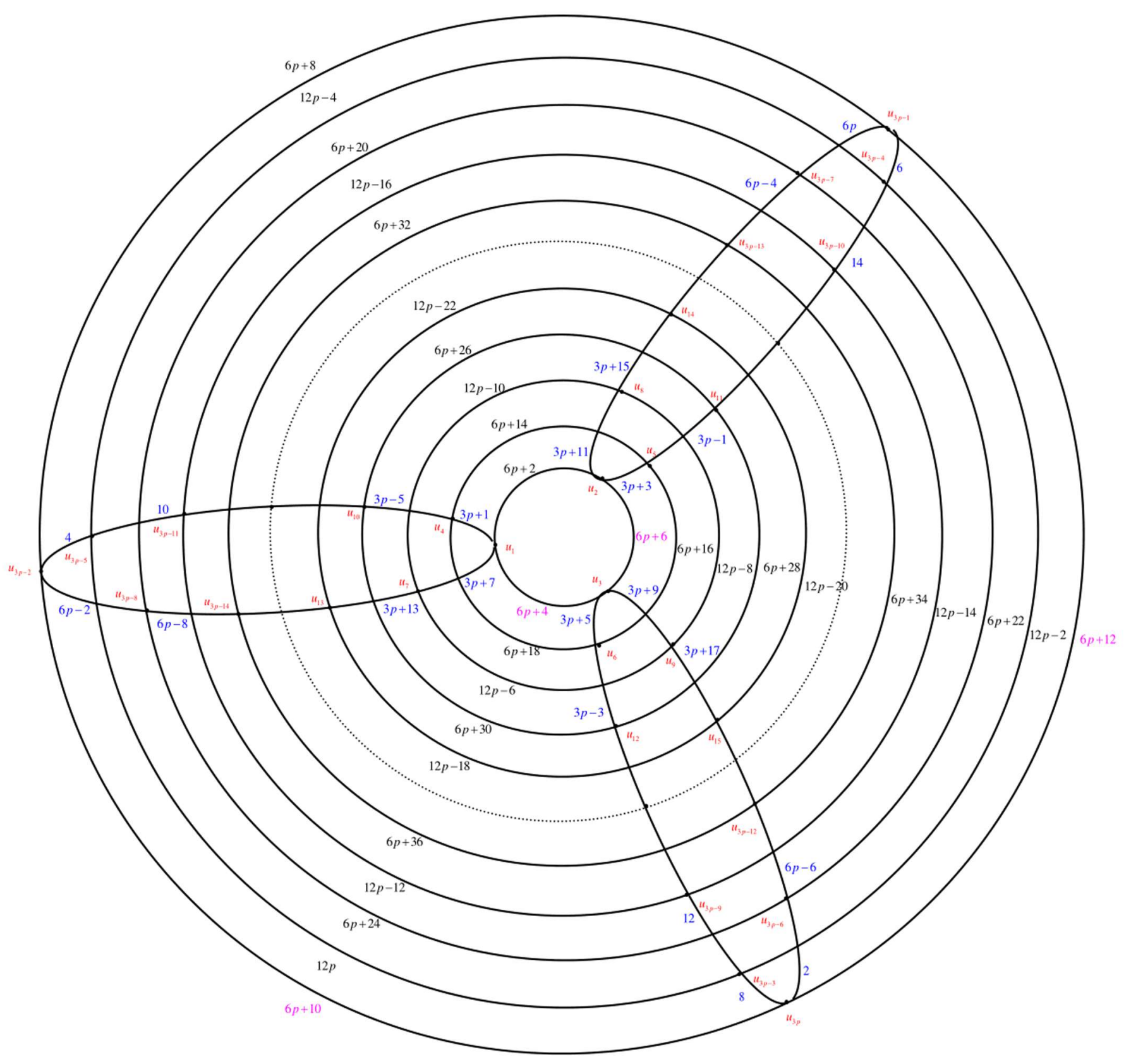

Figure 2.6: $T_{p, 3}, p>1, p$ is odd 
Illustration: The e. e. g. 1. of $T_{11,3}$ is presented in Figure 2.7.

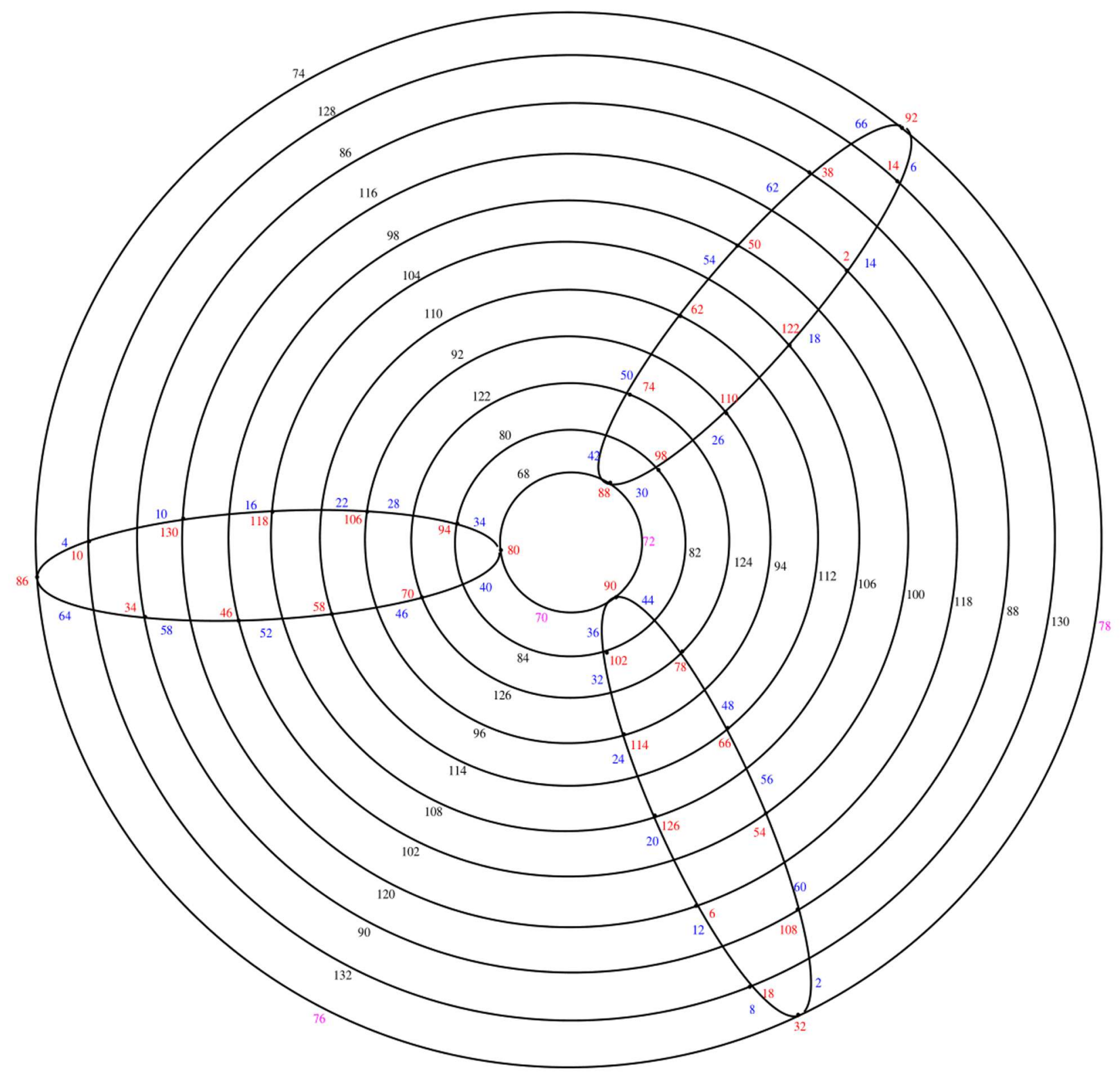

Figure 2.7: The e. e. g. l. of $T_{11,3}$ 
Lemma 2.0.4. Let $q=5$ and $p>1$ be an odd number that belongs to $\mathbf{Z}^{+}$. Then $T_{p, 5}$ is an e. e. g. graph.

Proof. Consider that $T_{p, 5}$, is given in Figure 2.8. First we label the edges of the ovals begin with the edges of the oval $C_{p}^{(1)}$ to the edges of the oval $C_{p}^{(5)}$ as follows:

Turn clockwise to label the edges

$u_{5 p-4} u_{5 p-9}, u_{5 p-3} u_{5 p-8}, u_{5 p-2} u_{5 p-7}, u_{5 p-1} u_{5 p-6}, u_{5 p} u_{5 p-5}$ by $2,4,8,10,12$, then turn counter-clockwise to label the edges

$u_{5 p-9} u_{5 p-19}, u_{5 p-5} u_{5 p-15}, u_{5 p-6} u_{5 p-16}, u_{5 p-7} u_{5 p-17}, u_{5 p} u_{5 p-18}$ by 14, 16, 18, 20,22 and so on.

Turn clockwise to label the edges

$u_{6} u_{1}, u_{7} u_{2}, u_{8} u_{3}, u_{9} u_{4}, u_{10} u_{5}$ by $5(p-3)+14,5(p-3)+16,5(p-3)+18,5(p-$ $3)+20,5(p-3)+22$,

then move clockwise to label the edges, then turn counter-clockwise to lable the edge

$u_{1} u_{11}, u_{5} u_{15}, u_{4} u_{14}, u_{3} u_{13}, u_{2} u_{12}$ by $5(p-3)+24,5(p-3)+26,5(p-3)+$ $28,5(p-3)+30,5(p-3)+32$ and so on. Finally turn clockwise to te edges $u_{5 q-14} u_{5 p-4}, u_{5 p-13} u_{5 p-3}, u_{5 p-12} u_{5 p-2}, u_{5 p-11} u_{5 p-1}, u_{5 p-10} u_{5 p}$ by $10(p-1)+$ $4,10(p-1)+6,10(p-1)+8,10 p, 6$.

Next, lable the edges of circles $C_{5}^{(p)}, 1 \leq j \leq p$ begin with the inner most circle $C_{5}^{(1)}$, then the edges of the circle $C_{5}^{(p)}$, then the edges of the circle $C_{5}^{(2)}$, then the edges of the circles $C_{5}^{(4)}, C_{5}^{(6)}, \cdots, C_{5}^{(p-1)}$, then the edges of the circles $C_{5}^{(3)}, C_{5}^{(5)}, \cdots, C_{5}^{(p-2)}$ as follows:

move clockwise to label the edges

$g\left(u_{1} u_{2}\right)=10 p+2 ; g\left(u_{2} u_{3}\right)=10 p+4 ; g\left(u_{3} u_{4}\right)=10 p+8 ; g\left(u_{4} u_{5}\right)=$ $10 p+6, g\left(u_{5} u_{1}\right)=10(p+1), g\left(u_{5 p+i-5} u_{5 p+i-4}\right)=10(p+1)+2 i, 1 \leq i \leq$ $4, g\left(u_{5 p} u_{5 q-4}\right)=10(p+2), g\left(u_{6} u_{7}\right)=10 p+22, g\left(u_{7} u_{8}\right)=10 p+26, g\left(u_{8} u_{9}\right)=$ $10 p+24, g\left(u_{9} u_{10}\right)=10 p+28, g\left(u_{10} u_{6}\right)=10(p+3), g\left(u_{5 j-5+i} u_{5 j-4+i}\right)=$ $10(p+j)+2 i, g\left(u_{5 j} u_{5 j-4}\right)=10(p+j+1), 1 \leq i \leq 4,4 \leq j \leq p-1$ where $j$ is even and

$$
g\left(u_{5 j-5+i} u_{5 j-4+i}\right)=10(2 p-j+1)+2 i, g\left(u_{5 j} u_{5 j-4}\right)=10(2 p-j+2),
$$
$1 \leq i \leq 4,3 \leq j \leq p-2$, where $j$ is odd.

Thus, the labels of the corresponding vertices mod $12 p$ become: $g^{*}\left(u_{1}\right) \equiv 10 p+20, g^{*}\left(u_{2}\right) \equiv 10 p+24, g^{*}\left(u_{3}\right) \equiv 10 p+30, g^{*}\left(u_{4}\right) \equiv 10 p+$ $32, g^{*}\left(u_{5}\right) \equiv 10 p+34 g^{*}\left(u_{5 p-4}\right) \equiv 10 p+28, g^{*}\left(u_{5 p-3}\right) \equiv 10 p+26, g^{*}\left(u_{5 p-2}\right) \equiv$ 
$10 p+36, g^{*}\left(u_{5 p-1}\right) \equiv 10 p+26, g^{*}\left(u_{5 p}\right) \equiv 56, g^{*}\left(u_{6}\right) \equiv 10 p+40, g^{*}\left(u_{7}\right) \equiv$ $10 p+46, g^{*}\left(u_{6}\right) \equiv 10 p+48, g^{*}\left(u_{9}\right) \equiv 10 p+50, g^{*}\left(u_{10}\right) \equiv 10 p+56, g^{*}\left(u_{5 j+i-5}\right) \equiv$ $10(p+j)+4 i+16,1 \leq i \leq 5,4 \leq j \leq p-3 j$ is even; $g^{*}\left(u_{5 j+i-5}\right) \equiv 10(p-$ $j)+4 i+26,1 \leq i \leq 5,3 \leq j \leq p-4, j$ is odd; $g^{*}\left(u_{5 p+4}\right) \equiv 50, g^{*}\left(u_{5 p-13}\right) \equiv$ $54, g^{*}\left(u_{5 p-12}\right) \equiv 58, g^{*}\left(u_{5 p-11}\right) \equiv 62, g^{*}\left(u_{5 p-10}\right) \equiv 10 p+70, g^{*}\left(u_{5 p-9}\right) \equiv$ $8, g^{*}\left(u_{5 p-8}\right) \equiv 12, g^{*}\left(u_{5 p-7}\right) \equiv 18, g^{*}\left(u_{5 p-6}\right) \equiv 22, g^{*}\left(u_{5 p-5}\right) \equiv 26$.

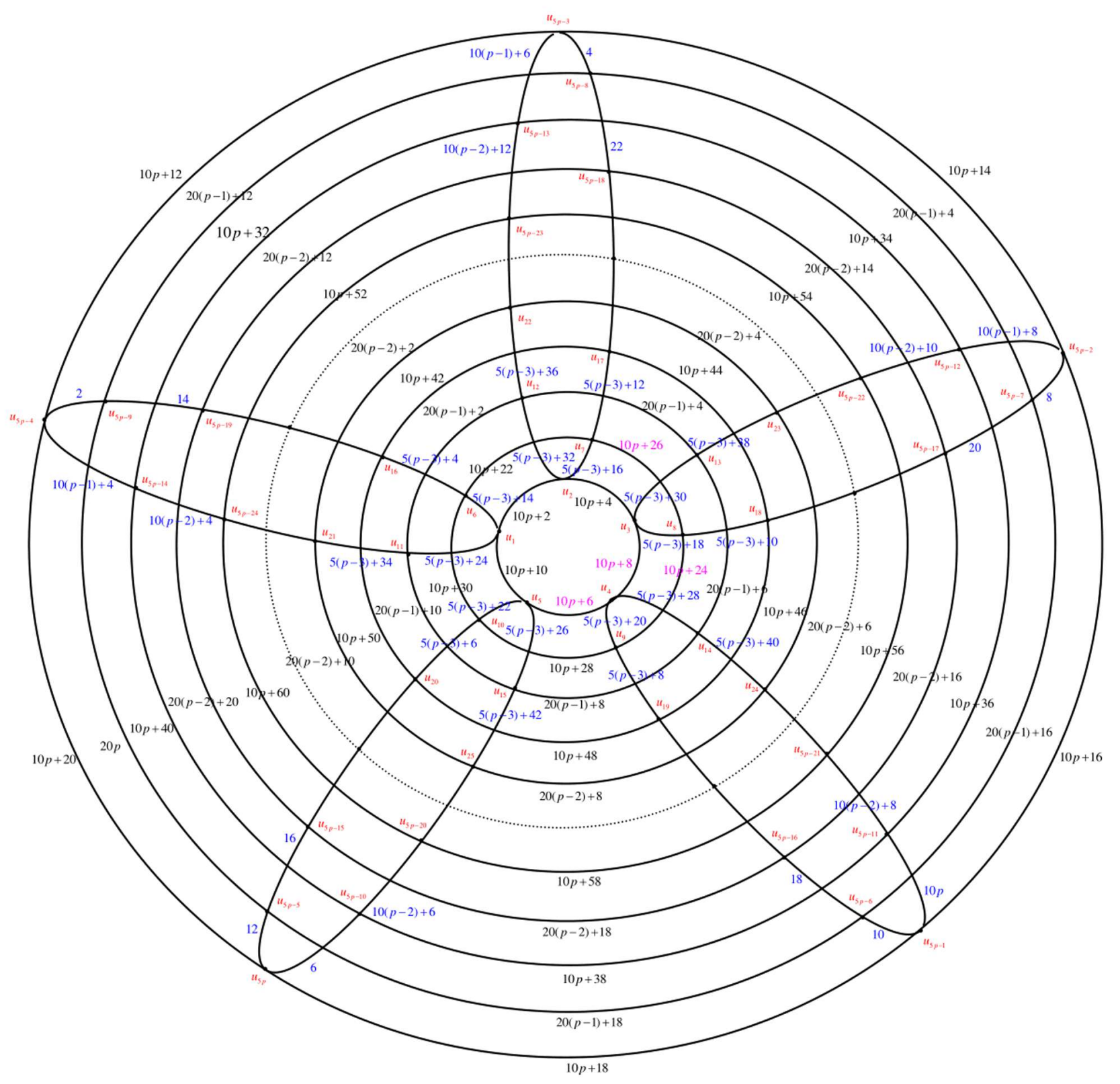

Figure 2.8: $T_{p, 5}, p>1, p$ is odd 
Illustration: The e. e. g. 1. of $T_{11,5}$ is presented in Figure 2.9.

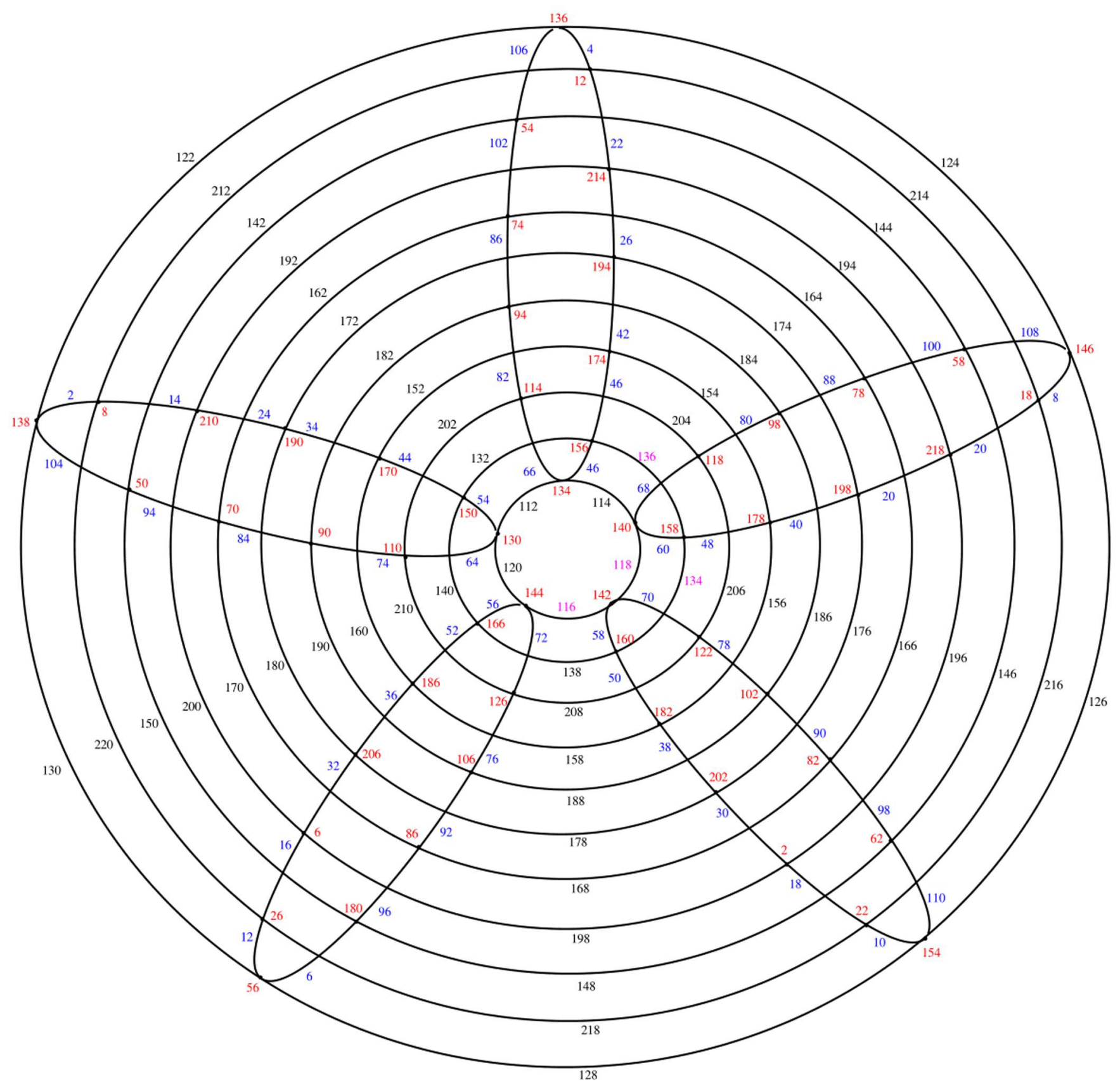

Figure 2.9: The e. e. g. l. of $T_{11,5}$ 
Lemma 2.0.5. Assume that $q>1$ is an odd number that belonges to $\mathbf{Z}^{+}$ and $p=3$. Then $T_{3, q}$ is an e. e. g. graph.

Proof. Consider that $T_{3, q}$ is given in Figure 2.10. As first step, we label the edges of the oval $C_{3}^{(j)}, 1 \leq j \leq q$ clockwise strating with the edges of the oval $C_{3}^{(1)}$ to the the edges of the oval $C_{3}^{(q)}$ as follows:

$$
g\left(u_{2 q+i} u_{q+i}\right)=2 i, g\left(u_{q+i} u_{i}\right)=2 q+2 i, g\left(u_{2 q+i} u_{i}\right)=4 q+2 i, 1 \leq i \leq n .
$$

Secondly, we label the edges of the circles $C_{q}^{(j)}, 1 \leq j \leq q$,respectively as follows: $g\left(u_{i} u_{i+1}\right)=6 q+2 i, g\left(u_{q} u_{1}\right)=8 q, g\left(u_{q+i} u_{q+i+1}\right)=10 q+2 i, g\left(u_{q} u_{1}\right)=$ $8 q, g\left(u_{2 q+i} u_{2 q+i+1}\right)=8 q+2 i, g\left(u_{q} u_{1}\right)=8 q, 1 \leq i \leq q-1$.

Thus the labels of the corresponding vertices $\bmod 12 q$ become: $g^{*}\left(u_{1}\right)=$ $8 q+6, g^{*}\left(u_{i}\right)=6 q+8 i-2, g^{*}\left(u_{q+1}\right)=6, g^{*}\left(u_{q+i}\right)=10 q+8 i-2, g^{*}\left(u_{2 q+1}\right)=$ $10 q+6, g^{*}\left(u_{2 q+i}\right)=8 q+8 i-2,2 \leq i \leq q$. 


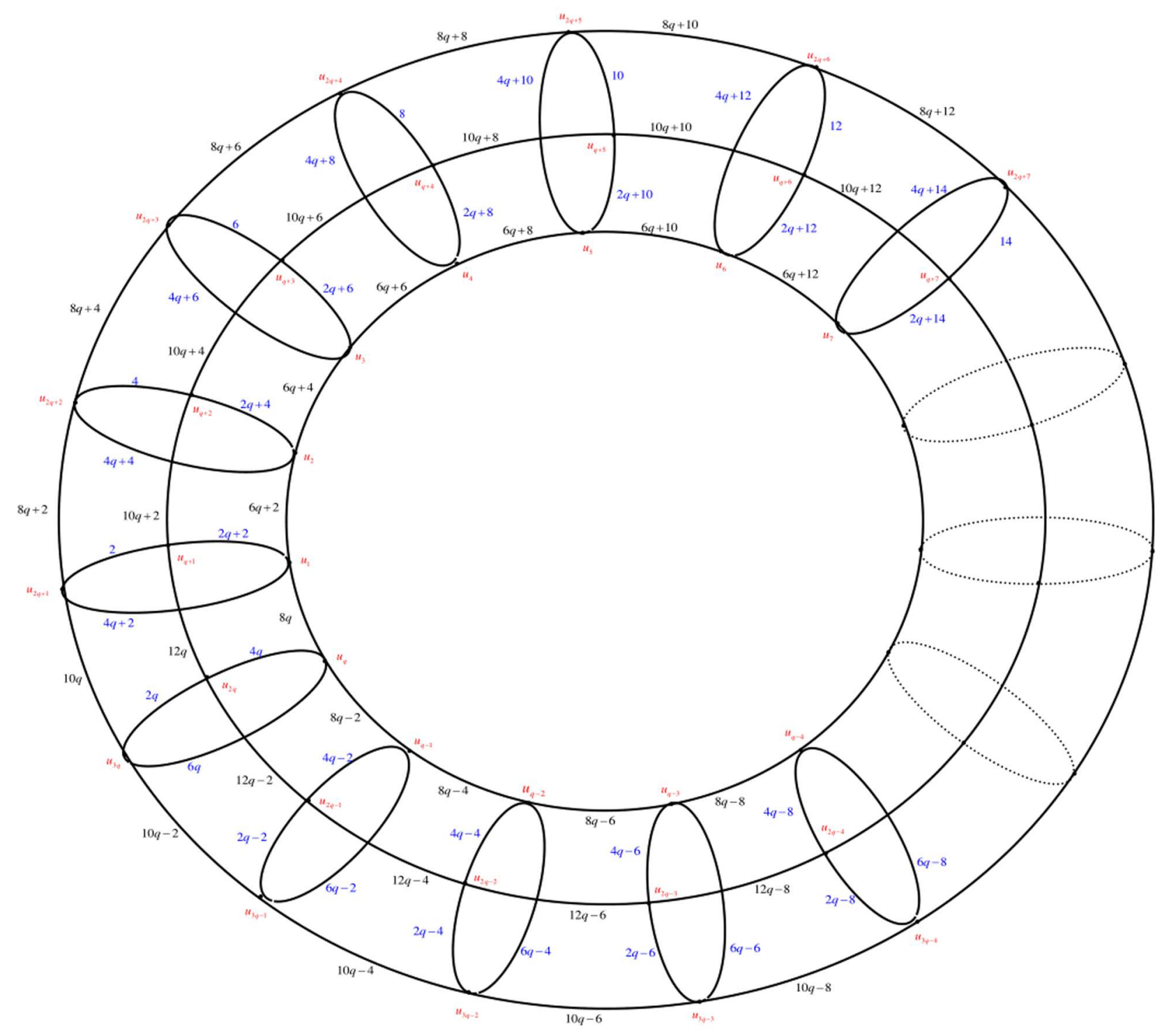

Figure 2.10: $T_{2, q}, q>1$ is odd positive. 
Illustration: The e. e. g. 1. of $T_{3,15}$ is given in Figure 2.11 .

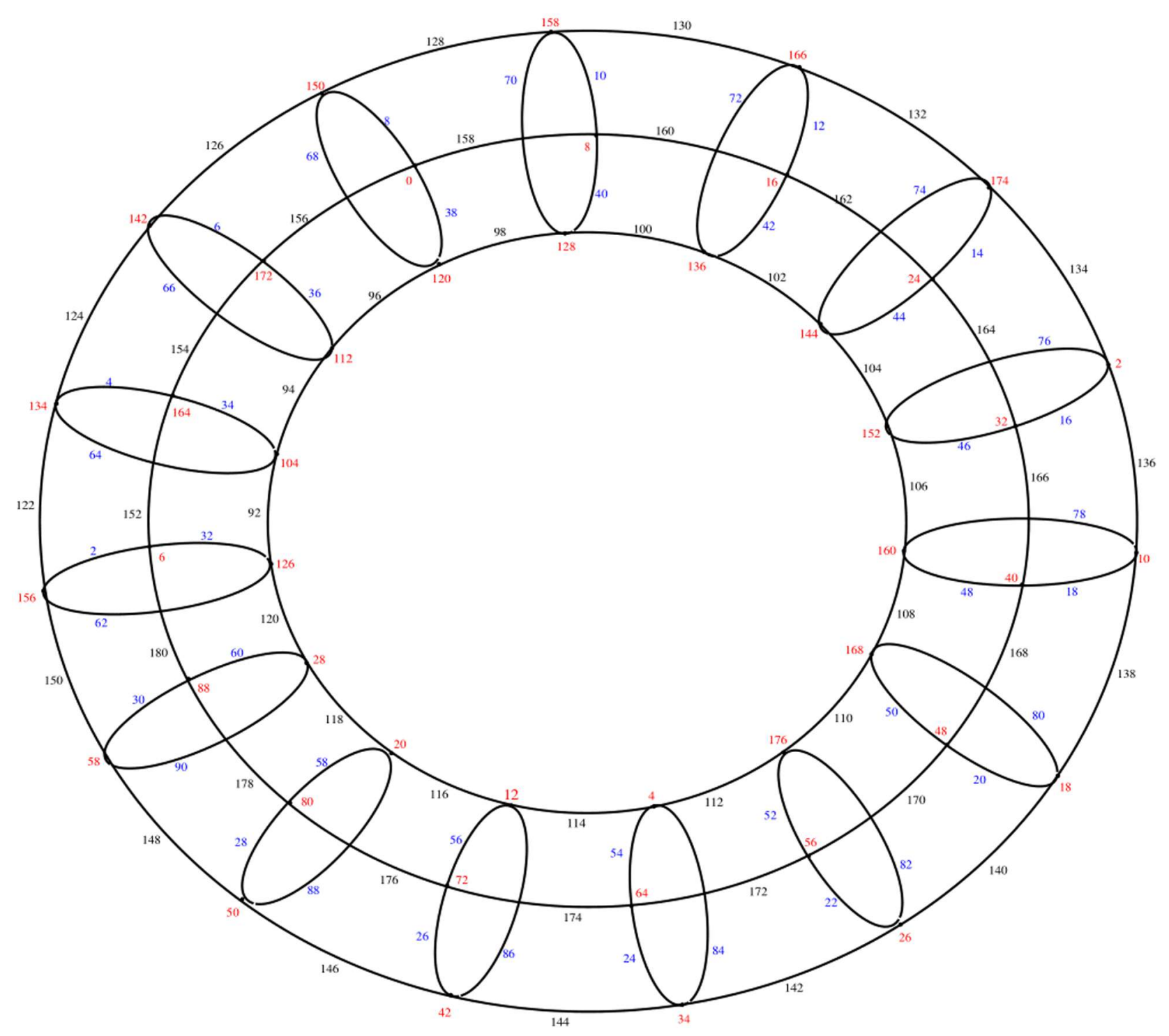

Figure 2.11: The e. e. g. l. of $T_{3,15}$ 
Theorem 2.0.6. Assume that $p>2$ is an even number that belongs to $\mathbf{Z}^{+}$and $q>1$ is an odd number that belongs to $\mathbf{Z}^{+}$. Then $T_{p, q}$ is an e. e. g. graph.

Proof. By using $n=\left|V\left(T_{p, q}\right)\right|=p q$ and $m=\left|E\left(T_{p, q}\right)\right|=2 p q$ and $r=\max \{m, n\}=2 p q$ and let $g: E\left(T_{p, q}\right) \longrightarrow\{2,4, \cdots, 4 p q-2\}$, hence there are four cases:

Case $1 q \equiv 0 \bmod 8$. Assume that $T_{p, q}$ is given as in Figure2.12. As first step, we label the edges of the ovals $C_{p}^{(j)}, 1 \leq j \leq q$ begin with the edges of the oval $C_{p}^{(1)}$ as follows:

we turn clockwise to label the edges

$$
\begin{array}{r}
u_{(p-1) q+1} u_{(p-2) q+1}, u_{(p-1) q+2} u_{(p-2) q+2}, u_{(p-1) q+3} u_{(p-2) q+3}, \\
\cdots, u_{p q+1} u_{(p-1) q-1}, u_{p q} u_{(p-1) q}
\end{array}
$$

by $2,4,6, \cdots, 2 q-2,2 q$, then we turn counter-clockewise to label the edges

$$
\begin{array}{r}
u_{(p-2) q+1} u_{(p-4) q+1}, u_{(p-1) q} u_{(p-3) q}, u_{(p-1) q-1} u_{(p-3) q-1}, \\
\cdots, u_{(p-2) q+3} u_{(p-4) q+3}, u_{(p-2) q+2} u_{(p-4) q+2}
\end{array}
$$

by $2 q+2,2 q+4,2 q+6, \cdots, 4 q-2,4 q$ and so on. If $p \equiv 1 \bmod 4$, we turn clockwise to label the edges $u_{q+1} u_{1}, u_{q+2} u_{2}, u_{q+3} u_{3}, \cdots, u_{2 q-1} u_{q-1}, u_{2 q} u_{q}$ by $(p-1) q+2,(p-1) q+4,(p-1) q+6, \cdots,(p+1) q-2,(p+1) q$, then we turn counter-clockwise to label the edges

$u_{1} u_{2 q+1}, u_{q} u_{3 q}, u_{q-1} u_{3 q-1}, \cdots, u_{3} u_{2 q+3}, u_{2} u_{2 n+2}$ by $(p+1) n+2,(p+1) q+$ $4,(p+1) q+6, \cdots,(p+3) q-2,(p+3) q$, next we turn clockwise to label the edges

$u_{2 q+1} u_{4 q+1}, u_{2 q+2} u_{4 q+2}, u_{2 q+3} u_{4 q+3}, \cdots, u_{3 q-1} u_{5 q-1}, u_{3 q} u_{5 q}$ by $(p+3) q+2,(p+$ 3) $q+4,(p+3) q+6, \cdots,(p+5) q-2,(p+5) q$ and so on. 


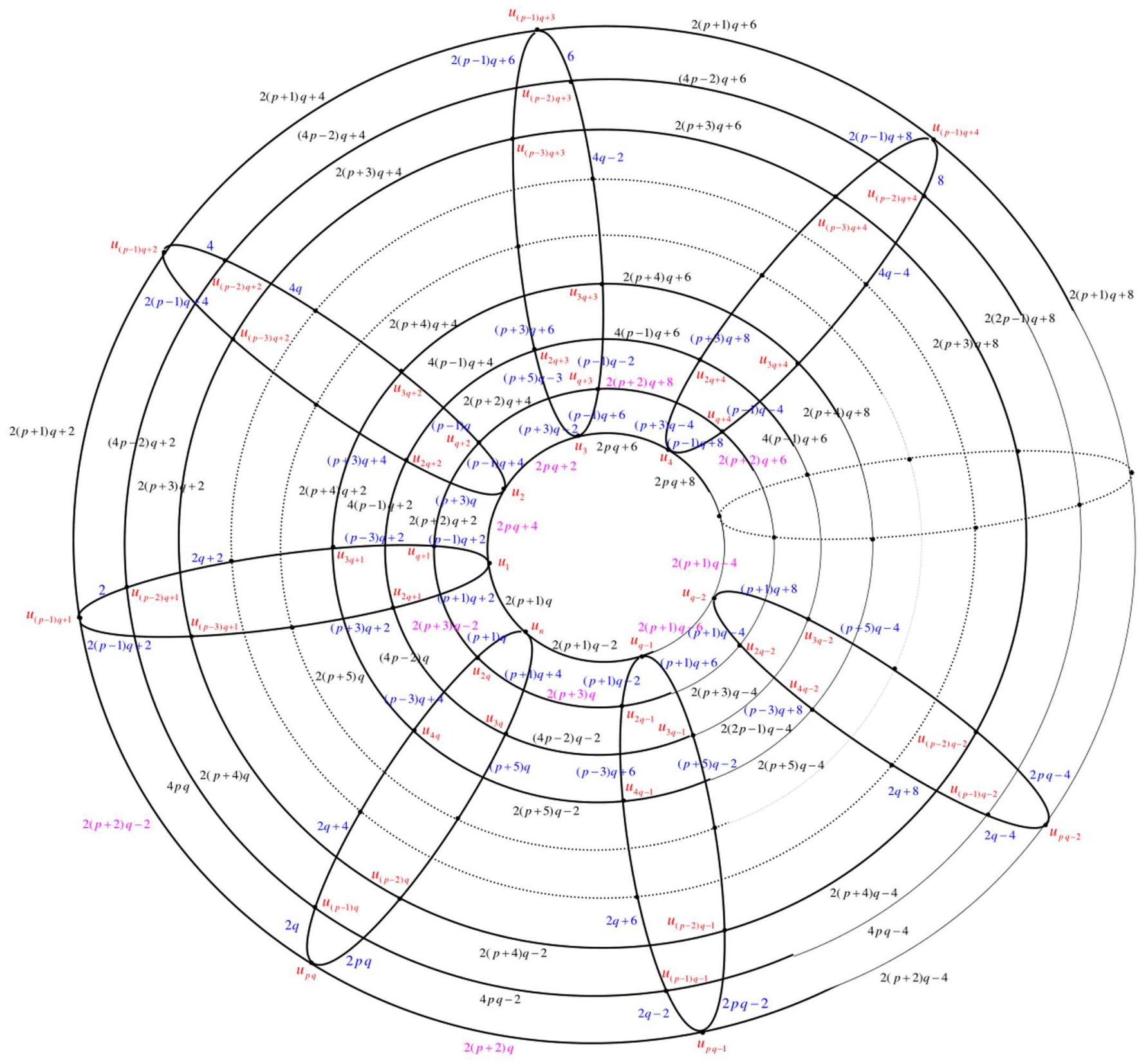

Figure 2.12: $T_{p, q}, p$ is even and $q \equiv 0 \bmod 8$ 
If $p \equiv 1 \bmod 4$, we turn counterm-clockwise to label the edges

$$
u_{q+1} u_{1}, u_{2 q} u_{q}, u_{2 q-1} u_{q-1}, \cdots, u_{q+3} u_{3}, u_{q+2} u_{2}
$$

by $(p-1) q+2,(p-1) q+4,(p-1) q+6, \cdots,(p+1) q-2,(p+1) q$, then we turn clockwise to label the edges $u_{1} u_{2 q+1}, u_{2} u_{2 q+2}, u_{3} u_{2 q+3}, \cdots, u_{q-1} u_{3 q-1}, u_{q} u_{3 q}$ by $(p+1) q+2,(p+1) q+4,(p+1) q+6, \cdots,(p+3) q-2,(p+3) q$, then we turn counter-clockwise anticlockwise to label the edges

$$
u_{2 q+1} u_{4 q+1}, u_{3 q} u_{5 q}, u_{3 q-1} u_{5 q-1}, \cdots, u_{2 q+3} u_{4 q+3}, u_{2 q+2} u_{4 q+2}
$$

by $(p+3) q+2,(p+3) q+4,(p+3) q+6, \cdots,(p+5) q-2,(p+5) q$ and so on.

Finally, we turn clockewise to label the edges

\section{Case 1}

$$
\begin{gathered}
u_{(p-3) q+1} u_{(p-1) q+1}, u_{(p-3) q+2} u_{(p-1) q+2}, u_{(p-3) q+3} u_{(p-1) q+3} \\
\cdots, u_{(p-2) q-1} u_{p q-1}, u_{(p-2) q} u_{p q}
\end{gathered}
$$

by $2 q(p-1)+2,2 q(p-1)+4,2 q(p-1)+6, \cdots, 2 p q-2,2 p q$. Second, we label the edges of the circles $C_{q}^{(j)}, 1 \leq j \leq p$ starting with the edges of the inner most circle $C_{q}^{(1)}$ as follows: $g\left(u_{1} u_{2}\right)=2 p q+4, g\left(u_{2} u_{3}\right)=3 p q+2, g\left(u_{3} u_{4}\right)=$ $2 p q+6, g\left(u_{4} u_{5}\right)=2 p q+8, g\left(u_{5} u_{6}\right)=2 p q+12, g\left(u_{6} u_{7}\right)=2 p q+10, g\left(u_{7} u_{8}\right)=$ $2 p q+14, g\left(u_{8} u_{9}\right)=2 p q+16, g\left(u_{9} u_{10}\right)=2 p q+20, g\left(u_{10} u_{11}\right)=2 p q+$ $18, g\left(u_{11} u_{12}\right)=2 p q+22, \cdots, g\left(u_{q-9} u_{q-8}\right)=2 q(p+1)-18, g\left(u_{q-8} u_{q-7}\right)=$ $2 q(p+1)-16, g\left(u_{q-7} u_{q-6}\right)=2 q(p+1)-12, g\left(u_{q-6} u_{q-5}\right)=2 q(p+1)-$ $14, g\left(u_{q-5} u_{q-4}\right)=2 q(p+1)-10, g\left(u_{q-4} u_{q-3}\right)=2 q(p+1)-8, g\left(u_{q-3} u_{q-2}\right)=$ $2 q(p+1)-4, g\left(u_{q-2} u_{q-1}\right)=2 q(p+1)-6, g\left(u_{q-1} u_{q}\right)=2 q(p+1)-2$ and $g\left(u_{q} u_{1}\right)=2 q(p+1)$, then label the edges of outer circle $C_{q}^{(p)}$ as follows: $g\left(u_{(p-1) q+1} u_{(p-1) q+i-1}\right)=2 q(p+1)+2 i, 1 \leq i \leq q-2, g\left(u_{q-1} u_{p q}\right)=$ $2 q(p+2)$ and $g\left(u_{p q} u_{q(p-1)-1}\right)=2 q(p+1)-2$ then we label the edges the circle $C_{q}^{(2)}$ as follows: $g\left(u_{q+1} u_{q+2}\right)=2 q(p+2)+2, g\left(u_{q+2} u_{q+3}\right)=$ $2 q(p+2)+4, g\left(u_{q+3} u_{q+4}\right)=2 q(p+2)+8, g\left(u_{q+4} u_{q+5}\right)=2 q(p+2)+$ $6, g\left(u_{q+5} u_{q+6}\right)=2 q(p+2)+10, g\left(u_{q+6} u_{q+7}\right)=2 q(p+2)+12, g\left(u_{q+7} u_{q+8}\right)=$ $2 q(p+2)+16, g\left(u_{q+8} u_{q+9}\right)=2 q(p+2)+14, g\left(u_{q+9} u_{q+10}\right)=2 q(p+2)+$ $18, g\left(u_{q+10} u_{q+11}\right)=2 q(p+2)+20, g\left(u_{q+11} u_{q+12}\right)=2 q(p+2)+24, \cdots, g\left(u_{2 q+9} u_{2 q+8}\right)=$ $2 q(p+3)-16, g\left(u_{2 q+8} u_{2 q+7}\right)=2 q(p+3)-18, g\left(u_{2 q+7} u_{2 q+6}\right)=2 q(p+$ $3)-14, g\left(u_{2 q+6} u_{2 q+5}\right)=2 q(p+3)-12, g\left(u_{2 q+5} u_{2 q+4}\right)=2 q(p+3)-$ $8, g\left(u_{2 q+4} u_{2 q+3}\right)=2 q(p+3)-10, g\left(u_{2 q+3} u_{2 q+2}\right)=2 q(p+3)-6, g\left(u_{2 q+2} u_{2 q+1}\right)=$ 
$2 q(p+3)-4, g\left(u_{2 q-1} u_{2 q}\right)=2 q(p+3)$ and $g\left(u_{2 q} u_{q+1}\right)=2 q(p+3)-$ 2. Next, we label the edges of circles $C_{q}^{(3)}, C_{q}^{(5)}, \cdots, C_{q}^{(p-2)}$ as follows: $g\left(u_{(j-1) q+i} u_{(j-1) q+i+1}\right)=4 q\left(p-\frac{j-1}{2}\right)+2 i$ and $g\left(u_{j q} u_{(j-1) q+1}\right)=4 q(p-$ $(2 j-4))-2,3 \leq j \leq p-2, j$ is odd and $1 \leq i \leq q-1$.

Finally, we label the edges of of the circles $C_{q}^{(4)}, C_{q}^{(6)}, \cdots, C_{q}^{(p-1)}$ as follows: $g\left(u_{(j-1) q+i} u_{(j-1) q+i+1}\right)=2 q(p+j)+2 i$ and $g\left(u_{j q} u_{(j-1) q+1}\right)=2 q(p+j+1)$, $4 \leq j \leq p-1, j$ is even and $1 \leq i \leq q-1$.

Thus the labels of the vertices of the inner post circle $C_{q}^{(1)} \bmod 4 p q$ are $g^{*}\left(u_{1}\right) \equiv 2 p q+2 q+8, g^{*}\left(u_{2}\right) \equiv 2 p q+2 q+10, g^{*}\left(u_{3}\right) \equiv 2 p q+2 q+12, g^{*}\left(u_{4}\right) \equiv$ $2 p q+2 q+18, g^{*}\left(u_{5}\right) \equiv 2 p q+2 q+24, g^{*}\left(u_{6}\right) \equiv 2 p q+2 q+26, g^{*}\left(u_{7}\right) \equiv$ $2 p q+2 q+28, g^{*}\left(u_{8}\right) \equiv 2 p q+2 q+34, g^{*}\left(u_{9}\right) \equiv 2 p q+2 q+40, g^{*}\left(u_{10}\right) \equiv$ $2 p q+2 q+42, g^{*}\left(u_{11}\right) \equiv 2 p q+2 q+44, g^{*}\left(u_{12}\right) \equiv 2 p q+2 q+50, \cdots, g^{*}\left(u_{q-8}\right) \equiv$ $2 p q+6 q-30, g^{*}\left(u_{q-7}\right) \equiv 2 p q+6 q-24, g^{*}\left(u_{q-6}\right) \equiv 2 p q+6 q-22, g^{*}\left(u_{q-5}\right) \equiv$ $2 p q+6 q-20, g^{*}\left(u_{q-4}\right) \equiv 2 p q+6 q-14, g^{*}\left(u_{q-3}\right) \equiv 2 p q+6 q-8, g^{*}\left(u_{q-2}\right) \equiv$ $2 p q+6 q-6, g^{*}\left(u_{q-1}\right) \equiv 2 p q+6 q-4$ and $g^{*}\left(u_{q}\right) \equiv 2 p q+6 q+2$.

The labels of the vertices of the outer circle $C_{q}^{(p)} \bmod 4 p q$ are: $g^{*}\left(u_{(p-1) q+1}\right) \equiv$ $2 p q+4 q+4, g^{*}\left(u_{(p-1) q+i}\right) \equiv 2 p q+2 q+8 i-2,2 \leq i \leq q-2 g^{*}\left(u_{p q-1}\right) \equiv$ $2 p q+10 q-8$ and $g^{*}\left(u_{p q}\right) \equiv 2 p q+10 q-2$ The labels of the vertices of the outer circle $C_{q}^{(2)} \bmod 4 p q$ become: $g^{*}\left(u_{q+1}\right) \equiv 2 p q+6 q+4, g^{*}\left(u_{q+2}\right) \equiv 2 p q+$ $6 q+10, g^{*}\left(u_{q+3}\right) \equiv 2 p q+6 q+16, g^{*}\left(u_{q+4}\right) \equiv 2 p q+6 q+18, g^{*}\left(u_{q+5}\right) \equiv 2 p q+$ $6 q+20, g^{*}\left(u_{q+6}\right) \equiv 2 p q+6 q+26, g^{*}\left(u_{q+7}\right) \equiv 2 p q+6 q+32, g^{*}\left(u_{q+8}\right) \equiv 2 p q+$ $6 q+34, g^{*}\left(u_{q+9}\right) \equiv 2 p q+6 q+36+g^{*}\left(u_{q+10}\right) \equiv 2 p q+6 q+42 \cdots, g^{*}\left(u_{2 q-8}\right) \equiv$ $2 p q+10 q-30, g^{*}\left(u_{2 q-7}\right) \equiv 2 p q+10 q-28, g^{*}\left(u_{2 q-6}\right) \equiv 2 p q+10 q-$ $22, g^{*}\left(u_{2 q-5}\right) \equiv 2 p q+10 q-16, g^{*}\left(u_{2 q-4}\right) \equiv 2 p q+10 q-14, g^{*}\left(u_{2 q-3}\right) \equiv$ $2 p q+10 q-12, g^{*}\left(u_{2 q-2}\right) \equiv 2 p q+10 q-6, g^{*}\left(u_{2 q-1}\right) \equiv 2 p q+10 q$ and $g^{*}\left(u_{2 q}\right) \equiv 2 p q+10 q+2$.

The labels of the vertices of circles $C_{q}^{(3)}, C_{q}^{(5)}, \cdots, C_{q}^{(p-2)} \bmod 4 p q$ become: $g^{*}\left(u_{j q+i}\right) \equiv 2 p q+2(j-1) q+4 i+2,2 \leq j \leq p-3, j$ is even and $1 \leq i \leq q$. The labels of the vertices of circles $C_{q}^{(4)}, C_{q}^{(6)}, \cdots, C_{q}^{(p-1)} \bmod 4 p q$ become: $g^{*}\left(u_{j q+i}\right) \equiv 2 p q+2(j+2) q+4 i+2,3 \leq j \leq p-2, j$ is odd and $1 \leq i \leq q$.

Case $2 q \equiv 2 \bmod 8$. Let $T_{p, q}$ be the torus grid graph that is given in Figure12. As first step, we label the edges of the ovals $C_{p}^{(j)}, 1 \leq j \leq n$ as the same in case (1).

Then, we label the edges of the circles $C_{q}^{(j)}, 1 \leq j \leq p$ begin with the edges of the inner most circle $C_{q}^{(1)}$ such as: $g\left(u_{1} u_{2}\right)=2 p q+2, g\left(u_{2} u_{3}\right)=$ $2 p q+4, g\left(u_{3} u_{4}\right)=2 p q+8, g\left(u_{4} u_{5}\right)=2 p q+6, g\left(u_{5} u_{6}\right)=2 p q+10, g\left(u_{6} u_{7}\right)=$ 
$2 p q+12, g\left(u_{7} u_{8}\right)=2 p q+16, g\left(u_{8} u_{9}\right)=2 p q+14, g\left(u_{9} u_{10}\right)=2 p q+18, g\left(u_{10} u_{11}\right)=$ $2 p q+20, g\left(u_{11} u_{12}\right)=2 p q+24, \cdots, g\left(u_{q-9} u_{q-8}\right)=2 q(p+1)-18, g\left(u_{q-8} u_{q-7}\right)=$ $2 q(p+1)-16, g\left(u_{q-7} u_{q-6}\right)=2 q(p+1)-12, g\left(u_{q-6} u_{q-5}\right)=2 q(p+1)-$ $14, g\left(u_{q-5} u_{q-4}\right)=2 q(p+1)-10, g\left(u_{q-4} u_{q-3}\right)=2 q(p+1)-8, g\left(u_{q-3} u_{q-2}\right)=$ $2 q(p+1)-4, g\left(u_{q-2} u_{q-1}\right)=2 q(p+1)-6, g\left(u_{q-1} u_{q}\right)=2 q(p+1)-2$ and $g\left(u_{q} u_{1}\right)=2 q(p+1)$.

Then we label the edges of outer circle $C_{q}^{(p)}$ as follows: $g\left(u_{(p-1) q+i} u_{q+3}\right)=$ $2 q(p+1)+2 i, 1 \leq i \leq q-2, g\left(u_{q-1} u_{p q}\right)=2 q(p+2)$ and $g\left(u_{p q} u_{q(p-1)+1}\right)=$ $2 q(p+2)-2$. Then, we label the edges the circle $C_{q}^{(2)}$ as follows: $g\left(u_{q+1} u_{q+2}\right)=$ $2 q(p+2)+2, g\left(u_{q+2} u_{q+3}\right)=2 q(p+2)+4, g\left(u_{q+3} u_{q+4}\right)=2 q(p+2)+$ $8, g\left(u_{q+4} u_{q+5}\right)=2 q(p+2)+6, g\left(u_{q+5} u_{q+6}\right)=2 q(p+2)+10, g\left(u_{q+6} u_{q+7}\right)=$ $2 q(p+2)+12, g\left(u_{q+7} u_{q+8}\right)=2 q(p+2)+16, g\left(u_{q+8} u_{q+9}\right)=2 q(p+2)+$ $14, g\left(u_{q+9} u_{q+10}\right)=2 q(p+2)+18, g\left(u_{q+10} u_{q+11}\right)=2 q(p+2)+20, g\left(u_{q+11} u_{q+12}\right)=$ $2 q(p+2)+24, \cdots, g\left(u_{2 q-9} u_{2 q-8}\right)=2 q(p+3)-18, g\left(u_{2 q-8} u_{2 q-7}\right)=2 q(p+$ $3)-16, g\left(u_{2 q-7} u_{2 q-6}\right)=2 q(p+3)-12, g\left(u_{2 q-6} u_{2 q-5}\right)=2 q(p+3)-$ $14, g\left(u_{2 q-5} u_{2 q-4}\right)=2 q(p+3)-10, g\left(u_{2 q-4} u_{2 q-3}\right)=2 q(p+3)-8, g\left(u_{2 q-3} u_{2 q-2}\right)=$ $2 q(p+3)-6, g\left(u_{2 q-2} u_{2 q-1}\right)=2 q(p+3)-4, g\left(u_{2 q-1} u_{2 q}\right)=2 n(p+3)$ and $g\left(u_{2 q} u_{q+1}\right)=2 q(p+3)-2$. Then we label the edges of circles $C_{q}^{(3)}, C_{q}^{(5)}, \cdots, C_{q}^{(p-2)}$ and $C_{q}^{(4)}, C_{q}^{(6)}, \cdots, C_{q}^{(p-1)} \bmod 4 p q$ as the same in case (1) .

Thus, the labels of the vertices of the inner most circle $C_{q}^{(1)} \bmod 4 p q$ are: $g^{*}\left(u_{1}\right) \equiv 2 p q+2 q+6, g^{*}\left(u_{2}\right) \equiv 2 p q+2 q+10, g^{*}\left(u_{3}\right) \equiv 2 p q+2 q+16, g^{*}\left(u_{4}\right) \equiv$ $2 p q+2 q+18, g^{*}\left(u_{5}\right) \equiv 2 p q+2 q+20, g^{*}\left(u_{6}\right) \equiv 2 p q+2 q+26, g^{*}\left(u_{7}\right) \equiv$ $2 p q+2 q+32, g^{*}\left(u_{8}\right) \equiv 2 p q+2 q+34, g^{*}\left(u_{9}\right) \equiv 2 p q+2 q+36, g^{*}\left(u_{10}\right) \equiv 2 p q+$ $2 q+42, \cdots, g^{*}\left(u_{q-8}\right) \equiv 2 p q+6 q-30, g^{*}\left(u_{q-7}\right) \equiv 2 p q+6 q-24, g^{*}\left(u_{q-6}\right) \equiv$ $2 p q+6 q-22, g^{*}\left(u_{q-5}\right) \equiv 2 p q+6 q-20, g^{*}\left(u_{q-4}\right) \equiv 2 p q+6 q-14, g^{*}\left(u_{q-3}\right) \equiv$ $2 p q+6 q-8, g^{*}\left(u_{q-2}\right) \equiv 2 p q+6 q-6, g^{*}\left(u_{q-1}\right) \equiv 2 p q+6 q-4$ and $g^{*}\left(u_{q}\right) \equiv$ $2 p q+6 q+2$.

The labels of the vertices of the outer circle $C_{q}^{(p)} \bmod 4 p q$ are as follows: $g^{*}\left(u_{(q-1) q+1}\right) \equiv 2 p q+4 q+4, g^{*}\left(u_{(p-1) q+i}\right) \equiv 2 p q+2 q+8 i-2,2 \leq i \leq$ $q-2, g^{*}\left(u_{p q-1}\right) \equiv 2 p q+10 q-8$ and $g^{*}\left(u_{p q}\right) \equiv 2 p q+10 q-2$.

The labels of the vertices of the circle $C_{q}^{(2)} \bmod 4 p q$ are as follows: $g^{*}\left(u_{q+1}\right) \equiv$ $2 p q+6 q+4, g^{*}\left(u_{q+2}\right) \equiv 2 p q+6 q+10, g^{*}\left(u_{q+3}\right) \equiv 2 p q+6 q+16, g^{*}\left(u_{q+4}\right) \equiv$ $2 p q+6 q+18, g^{*}\left(u_{q+5}\right) \equiv 2 p q+6 q+20, g^{*}\left(u_{q+6}\right) \equiv 2 p q+6 q+26, g^{*}\left(u_{q+7}\right) \equiv$ $2 p q+6 q+32, g^{*}\left(u_{q+8}\right) \equiv 2 p q+6 q+34, g^{*}\left(u_{q+9}\right) \equiv 2 p q+6 q+36, g^{*}\left(u_{q+10}\right) \equiv$ $2 p q+6 q+42 \cdots, g^{*}\left(u_{2 q-8}\right) \equiv 2 p q+10 q-30, g^{*}\left(u_{2 q-7}\right) \equiv 2 p q+10 q-$ $24, g^{*}\left(u_{2 q-6}\right) \equiv 2 p q+10 q-22, g^{*}\left(u_{2 q-5}\right) \equiv 2 p q+10 q-20, g^{*}\left(u_{2 q-4}\right) \equiv 2 p q+$ $10 q-14, g^{*}\left(u_{2 q-3}\right) \equiv 2 p q+10 q-10, g^{*}\left(u_{2 q-2}\right) \equiv 2 p q+10 q-6, g^{*}\left(u_{2 q-1}\right) \equiv$ $2 p q+10 q$ and $g^{*}\left(u_{2 p}\right) \equiv 2 p q+10 q+2$. 
The labels of the vertices of circles $C_{q}^{(3)}, C_{q}^{(5)}, \cdots, C_{q}^{(p-2)}$ and $C_{q}^{(4)}, C_{q}^{(6)}, \cdots, C_{q}^{(p-1)} \bmod 4 p q$ are the same as in case (1).

Case $3 q \equiv 4 \bmod 8$. Assume that $T_{p, q}$ is given in Figure2.12. First, we label the edges of the ovals $C_{p}^{(j)}, 1 \leq j \leq q$ as the same in case (1). Secondly, we label the edges of the circles $C_{q}^{(j)}, 1 \leq j \leq p$ begin with the edges of the inner most circle $C_{q}^{(1)}$ as follows: $g\left(u_{1} u_{2}\right)=2 p q+4, g\left(u_{2} u_{3}\right)=2 p q+2, g\left(u_{3} u_{4}\right)=$ $2 p q+6, g\left(u_{4} u_{5}\right)=2 p q+8, g\left(u_{5} u_{6}\right)=2 p q+12, g\left(u_{6} u_{7}\right)=2 p q+10, g\left(u_{7} u_{8}\right)=$ $2 p q+14, g\left(u_{8} u_{9}\right)=2 p q+16, g\left(u_{9} u_{10}\right)=2 p q+20, g\left(u_{10} u_{11}\right)=2 p q+$ $18, g\left(u_{11} u_{12}\right)=2 p q+22, \cdots, g\left(u_{q-9} u_{q-8}\right)=2 q(p+1)-18, g\left(u_{q-8} u_{q-7}\right)=$ $2 q(p+1)-16, g\left(u_{q-7} u_{q-6}\right)=2 q(p+1)-12, g\left(u_{q-6} u_{q-5}\right)=2 q(p+1)-$ $14, g\left(u_{q-5} u_{q-4}\right)=2 q(p+1)-10, g\left(u_{q-4} u_{q-3}\right)=2 q(p+1)-8, g\left(u_{q-3} u_{q-2}\right)=$ $2 q(p+1)-4, g\left(u_{q-2} u_{q-1}\right)=2 q(p+1)-6, g\left(u_{q-1} u_{q}\right)=2 q(p+1)-2$ and $g\left(u_{q} u_{1}\right)=2 q(p+1)$. Hence the edges of outer circle $C_{q}^{(p)}$ are labeled as: $g\left(u_{(p-1) q+1} u_{(p-1) q+2}\right)=4, g\left(u_{(p-1) q+2} u_{(p-1) q+3}\right)=2 q(p+1)+$ $2, g\left(u_{(p-1) q+i} u_{(p-1) q+i+1}\right)=2 q(p+1)+2 i, 3 \leq i \leq q-2$ and $g\left(u_{p q} u_{q(p-1)+1}\right)=$ $2 q(p+2)$. Then we label the edges the circle $C_{q}^{(2)}$ as follows: $g\left(u_{q+1} u_{q+2}\right)=$ $2 q(p+2)+2, g\left(u_{q+2} u_{q+3}\right)=2 q(p+2)+4, g\left(u_{q+3} u_{q+4}\right)=2 q(p+2)+$ $8, g\left(u_{q+4} u_{q+5}\right)=2 q(p+2)+6, g\left(u_{q+5} u_{q+6}\right)=2 q(p+2)+10, g\left(u_{q+6} u_{q+7}\right)=$ $2 q(p+2)+12, g\left(u_{q+7} u_{q+8}\right)=2 q(p+2)+16, g\left(u_{q+8} u_{q+9}\right)=2 q(p+2)+$ $14, g\left(u_{q+9} u_{q+10}\right)=2 q(p+2)+18, g\left(u_{q+10} u_{q+11}\right)=2 q(p+2)+20, \cdots, g\left(u_{2 q-9} u_{2 q-8}\right)=$ $2 q(p+3)-16, g\left(u_{2 q-8} u_{2 q-7}\right)=2 q(p+3)-18, g\left(u_{2 q-7} u_{2 q-6}\right)=2 q(p+$ $3)-14, g\left(u_{2 q-6} u_{2 q-5}\right)=2 q(p+3)-12, g\left(u_{2 q-5} u_{2 q-4}\right)=2 q(p+3)-$ $8, g\left(u_{2 q-4} u_{2 q-3}\right)=2 q(p+3)-10, g\left(u_{2 q-3} u_{2 q-2}\right)=2 q(p+3)-6, g\left(u_{2 q-2} u_{2 q-1}\right)=$ $2 q(p+3)-4, g\left(u_{2 q-1} u_{2 q}\right)=2 q(p+3)$ and $g\left(u_{2 q} u_{q+1}\right)=2 q(p+3)-2$.

Then we label the edges of circles $C_{q}^{(3)}, C_{q}^{(5)}, \cdots, C_{q}^{(p-2)}$ and $C_{q}^{(4)}, C_{q}^{(6)}, \cdots, C_{q}^{(p-1)} \bmod 4 p q$ as the same in case (1).

Thus, the labels of the vertices of the inner most circle $C_{q}^{(1)} \bmod 4 p q$ are: $g^{*}\left(u_{1}\right) \equiv 2 p q+2 q+8, g^{*}\left(u_{2}\right) \equiv 2 p q+2 q+10, g^{*}\left(u_{3}\right) \equiv 2 p q+2 q+12, g^{*}\left(u_{4}\right) \equiv$ $2 p q+2 q+18, g^{*}\left(u_{5}\right) \equiv 2 p q+2 q+24, g^{*}\left(u_{6}\right) \equiv 2 p q+2 q+26, g^{*}\left(u_{7}\right) \equiv$ $2 p q+2 q+28, g^{*}\left(u_{8}\right) \equiv 2 p q+2 q+34, g^{*}\left(u_{9}\right) \equiv 2 p q+2 q+40, g^{*}\left(u_{10}\right) \equiv$ $2 p q+2 q+42, g^{*}\left(u_{11}\right) \equiv 2 p q+2 q+44, g^{*}\left(u_{12}\right) \equiv 2 p q+2 q+50, \cdots, g^{*}\left(u_{q-8}\right) \equiv$ $2 p q+6 q-30, g^{*}\left(u_{q-7}\right) \equiv 2 p q+6 q-24, g^{*}\left(u_{q-6}\right) \equiv 2 p q+6 q-22, g^{*}\left(u_{q-5}\right) \equiv$ $2 p q+6 q-20, g^{*}\left(u_{q-4}\right) \equiv 2 p q+6 q-14, g^{*}\left(u_{q-3}\right) \equiv 2 p q+6 q-8, g^{*}\left(u_{q-2}\right) \equiv$ $2 p q+6 q-6, g^{*}\left(u_{q-1}\right) \equiv 2 p q+6 q-4$ and $g^{*}\left(u_{q}\right) \equiv 2 p q+6 q+2$.

The labels of the vertices of the outer circle $C_{q}^{(2)} \bmod 4 p q$ are as follows: $g^{*}\left(u_{q+1}\right) \equiv 2 p q+6 q+4, g^{*}\left(u_{q+2}\right) \equiv 2 p q+6 q+10, g^{*}\left(u_{q+3}\right) \equiv 2 p q+$ $6 q+16, g^{*}\left(u_{q+4}\right) \equiv 2 p q+6 q+18, g^{*}\left(u_{q+5}\right) \equiv 2 p q+6 q+20, g^{*}\left(u_{q+6}\right) \equiv$ 
$2 p q+6 q+26, g^{*}\left(u_{q+7}\right) \equiv 2 p q+6 q+32, g^{*}\left(u_{q+8}\right) \equiv 2 p q+6 q+34, g^{*}\left(u_{q+9}\right) \equiv$ $2 p q+6 q+36, g^{*}\left(u_{q+10}\right) \equiv 2 p q+6 q+42, \cdots, g^{*}\left(u_{2 q-8}\right) \equiv 2 p q+10 q-$ $30, g^{*}\left(u_{2 q-7}\right) \equiv 2 p q+10 q-28, g^{*}\left(u_{2 q-6}\right) \equiv 2 p q+10 q-22, g^{*}\left(u_{2 q-5}\right) \equiv 2 p q+$ $10 q-16, g^{*}\left(u_{2 q-4}\right) \equiv 2 p q+10 q-14, g^{*}\left(u_{2 q-3}\right) \equiv 2 p q+10 q-12, g^{*}\left(u_{2 q-2}\right) \equiv$ $2 p q+10 q-6, g^{*}\left(u_{2 q-1}\right) \equiv 2 p q+10 q, g^{*}\left(u_{2 q}\right) \equiv 2 p q+10 q+2$.

The labels of the vertices of circles $C_{q}^{(3)}, C_{q}^{(5)}, \cdots, C_{q}^{(p-2)}$ and $C_{q}^{(4)}, C_{q}^{(6)}, \cdots, C_{q}^{(p-1)} \bmod 4 p q$ as the same in case $(1)$.

Case $4 q \equiv 6 \bmod 8$. Let $T_{p, q}$ be such as in Figure 2.12. First, we label the edges of the ovals $C_{p}^{(j)}, 1 \leq j \leq q$ as the same in case (1).

Secondly we label the edges of the circles $C_{q}^{(j)}, 1 \leq j \leq p$ begin with the edges of the inner most circle $C_{q}^{(1)}$ as follows: $g\left(u_{1} u_{2}\right)=2 p q+4, g\left(u_{2} u_{3}\right)=$ $2 p q+2, g\left(u_{3} u_{4}\right)=2 p q+6, g\left(u_{4} u_{5}\right)=2 p q+8, g\left(u_{5} u_{6}\right)=2 p q+12, g\left(u_{6} u_{7}\right)=$ $2 p q+10, g\left(u_{7} u_{8}\right)=2 p q+14, g\left(u_{8} u_{9}\right)=2 p q+16, g\left(u_{9} u_{10}\right)=2 p q+20, g\left(u_{10} u_{11}\right)=$ $2 p q+18, g\left(u_{11} u_{12}\right)=2 p q+22, \cdots, g\left(u_{q-9} u_{q-8}\right)=2 q(p+1)-18, g\left(u_{q-8} u_{q-7}\right)=$ $2 q(p+1)-16, g\left(u_{q-7} u_{q-6}\right)=2 q(p+1)-12, g\left(u_{q-6} u_{q-5}\right)=2 q(p+1)-$ $14, g\left(u_{q-5} u_{q-4}\right)=2 q(p+1)-10, g\left(u_{q-4} u_{q-3}\right)=2 q(p+1)-8, g\left(u_{q-3} u_{q-2}\right)=$ $2 q(p+1)-4, g\left(u_{q-2} u_{q-1}\right)=2 q(p+1)-6, g\left(u_{q-1} u_{q}\right)=2 q(p+1)$ and $g\left(v_{q} v_{1}\right)=2 q(p+1)-2$. Next the edges of outer circle $C_{q}^{(p)}$ are labeled as: $g\left(u_{(p-1) q+1} u_{(p-1) q+i+1}\right)=2 q(p+1)+2 i, 1 \leq q \leq q-2, g\left(u_{q-1} u_{p q}\right)=$ $2 q(p+2), g\left(u_{p q} u_{q(p-1)+1}\right)=2 q(p+2)-2$.

Then, we label the edges the circle $C_{q}^{(2)}$ as follows: $g\left(u_{q+1} u_{q+2}\right)=$ $2 q(p+2)+4, g\left(u_{q+2} u_{q+3}\right)=2 q(p+2)+2, g\left(u_{q+3} u_{q+4}\right)=2 q(p+2)+$ $6, g\left(u_{q+4} u_{q+5}\right)=2 q(p+2)+8, g\left(u_{q+5} u_{q+6}\right)=2 q(p+2)+12, g\left(u_{q+6} u_{q+7}\right)=$ $2 q(p+2)+10, g\left(u_{q+7} u_{q+8}\right)=2 q(p+2)+16, g\left(u_{q+8} u_{q+9}\right)=2 q(p+2)+$ $14, g\left(u_{q+9} u_{q+10}\right)=2 q(p+2)+20, g\left(u_{q+10} u_{q+11}\right)=2 q(p+2)+18, \cdots, g\left(u_{2 q-9} u_{2 q-8}\right)=$ $2 q(p+3)-16, g\left(u_{2 q-8} u_{2 q-7}\right)=2 q(p+3)-18, g\left(u_{2 q-7} u_{2 q-6}\right)=2 q(p+$ $3)-14, g\left(u_{2 q-6} u_{2 q-5}\right)=2 q(p+3)-12, g\left(u_{2 q-5} u_{2 q-4}\right)=2 q(p+3)-$ $8, g\left(u_{2 q-4} u_{2 q-3}\right)=2 q(p+3)-10, g\left(u_{2 q-3} u_{2 q-2}\right)=2 q(p+3)-4, g\left(u_{2 q-2} u_{2 q-1}\right)=$ $2 q(p+3)-6, g\left(u_{2 q-1} u_{2 q}\right)=2 q(p+3)-2$ and $g\left(u_{2 q} u_{q+1}\right)=2 q(p+3)$.

Then we label the edges of circles $C_{q}^{(3)}, C_{q}^{(5)}, \cdots, C_{q}^{(p-2)}$ and $C_{q}^{(4)}, C_{q}^{(6)}, \cdots, C_{q}^{(p-1)} \bmod 4 p q$ as the same in case $(1)$.

Thus, the labels of the vertices of the inner most circle $C_{q}^{(1)} \bmod 4 p q$ are given as: $g^{*}\left(u_{1}\right) \equiv 2 p q+2 q+6, g^{*}\left(u_{2}\right) \equiv 2 p q+2 q+10, g^{*}\left(u_{3}\right) \equiv$ $2 p q+2 q+12, g^{*}\left(u_{4}\right) \equiv 2 p q+2 q+18, g^{*}\left(u_{5}\right) \equiv 2 p q+2 q+24, g^{*}\left(u_{6}\right) \equiv$ $2 p q+2 q+26, g^{*}\left(u_{7}\right) \equiv 2 p q+2 q+28, g^{*}\left(u_{8}\right) \equiv 2 p q+2 q+34, g^{*}\left(u_{9}\right) \equiv$ $2 p q+2 q+40, g^{*}\left(u_{10}\right) \equiv 2 p q+2 q+42, g^{*}\left(u_{11}\right) \equiv 2 p q+2 q+44, g^{*}\left(u_{12}\right) \equiv 2 p q+$ 
$2 q+50, \cdots, g^{*}\left(u_{q-8}\right) \equiv 2 p q+6 q-30, g^{*}\left(u_{q-7}\right) \equiv 2 p q+6 q-28, g^{*}\left(u_{q-6}\right) \equiv$ $2 p q+6 q-22, g^{*}\left(u_{q-5}\right) \equiv 2 p q+6 q-16, g^{*}\left(u_{q-4}\right) \equiv 2 p q+6 q-14, g^{*}\left(u_{q-3}\right) \equiv$ $2 p q+6 q-12, g^{*}\left(u_{q-2}\right) \equiv 2 p q+6 q-6, g^{*}\left(u_{q-1}\right) \equiv 2 p q+6 q$ and $g^{*}\left(u_{q}\right) \equiv$ $2 p q+6 q+2$.

The labels of the vertices of the outer circle $C_{q}^{(p)} \bmod 4 p q$ are the same in case (1).

The labels of the vertices of the circle $C_{q}^{(2)} \bmod 4 p q$ are:

$g^{*}\left(u_{q+1}\right) \equiv 2 p q+6 q+8, g^{*}\left(u_{q+2}\right) \equiv 2 p q+6 q+10, g^{*}\left(u_{q+3}\right) \equiv 2 p q+$ $6 q+12, g^{*}\left(u_{q+4}\right) \equiv 2 p q+6 q+18, g^{*}\left(u_{q+5}\right) \equiv 2 p q+6 q+24, g^{*}\left(u_{q+6}\right) \equiv$ $2 p q+6 q+26, g^{*}\left(u_{q+7}\right) \equiv 2 p q+6 q+28, g^{*}\left(u_{q+8}\right) \equiv 2 p q+6 q+34, g^{*}\left(u_{q+9}\right) \equiv$ $2 p q+6 q+40, g^{*}\left(u_{q+10}\right) \equiv 2 p q+6 q+42, \cdots, g^{*}\left(u_{2 q-8}\right) \equiv 2 p q+10 q-$ $30, g^{*}\left(u_{2 q-7}\right) \equiv 2 p q+10 q-28, g^{*}\left(u_{2 q-6}\right) \equiv 2 p q+10 q-22, g^{*}\left(u_{2 q-5}\right) \equiv 2 p q+$ $10 q-16, g^{*}\left(u_{2 q-4}\right) \equiv 2 p q+10 q-14, g^{*}\left(u_{2 q-3}\right) \equiv 2 p q+10 q-10, g^{*}\left(u_{2 q-2}\right) \equiv$ $2 p q+10 q-6, g^{*}\left(u_{2 q-1}\right) \equiv 2 p q+10 q-4, g^{*}\left(u_{2 q}\right) \equiv 2 p q+10 q+2$.

The labels of the verticesof circles $C_{q}^{(3)}, C_{q}^{(5)}, \cdots, C_{q}^{(p-2)}$ and $C_{q}^{(4)}, C_{q}^{(6)}, \cdots, C_{q}^{(p-1)} \bmod 4 p q$ as the same in case (1). 
Illustration: The e. e. g. 1 . of $T_{8,9}, T_{10,9}, T_{12,9}$ and $T_{14,9}$ are presented in Figure 2.13, respectively.

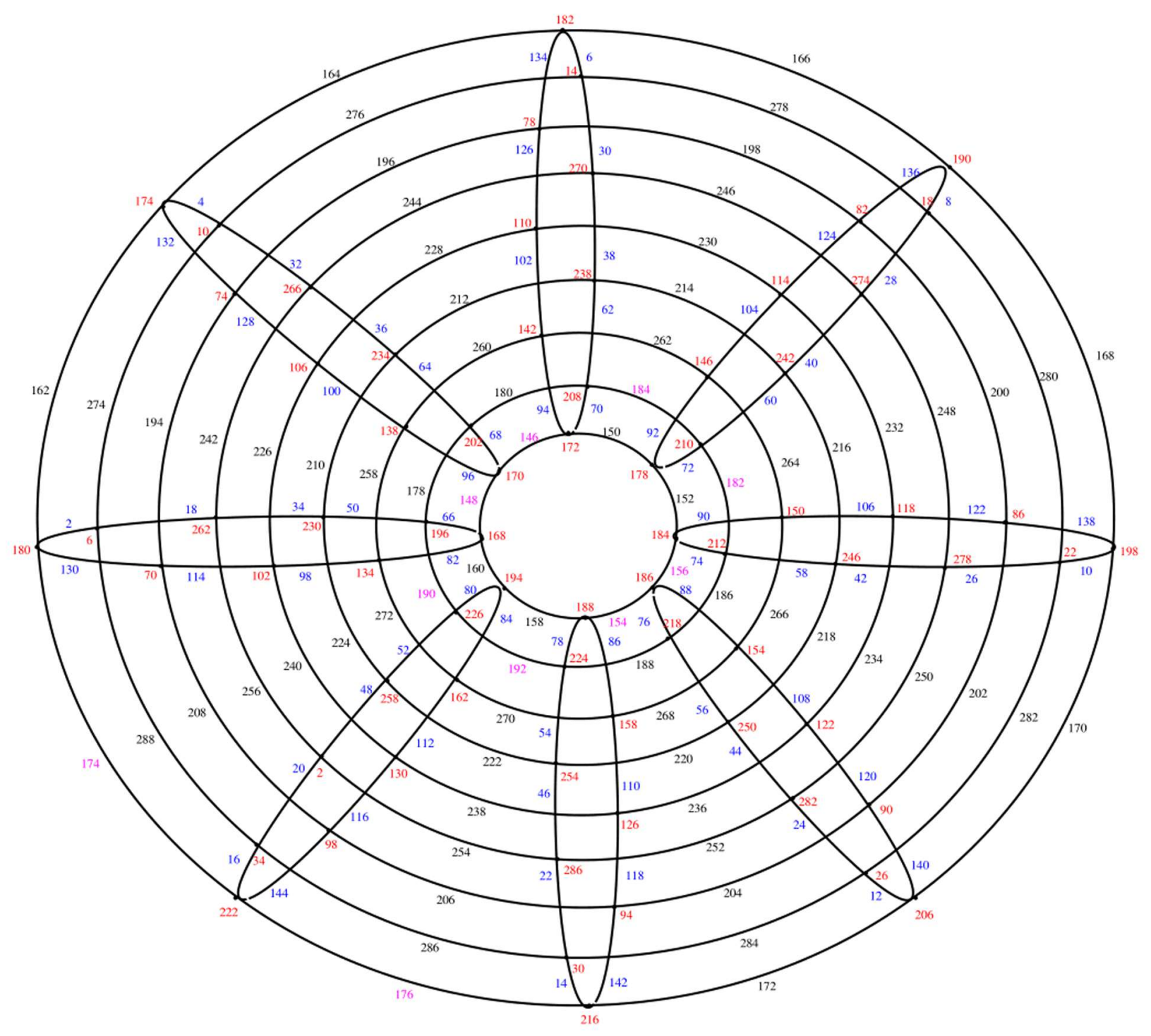

(a) $T_{8,9}$ 


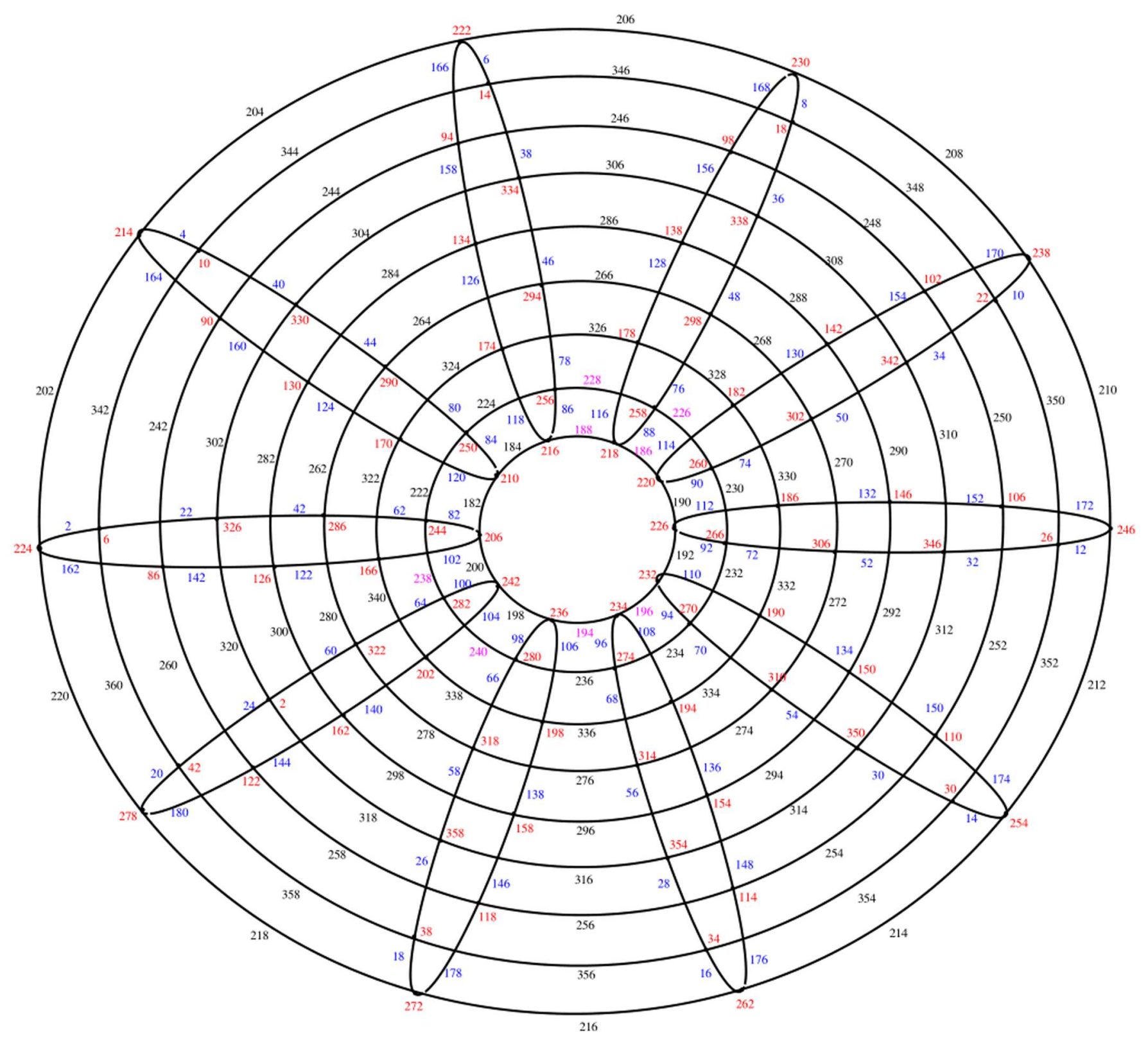

(b) $T_{10,9}$ 


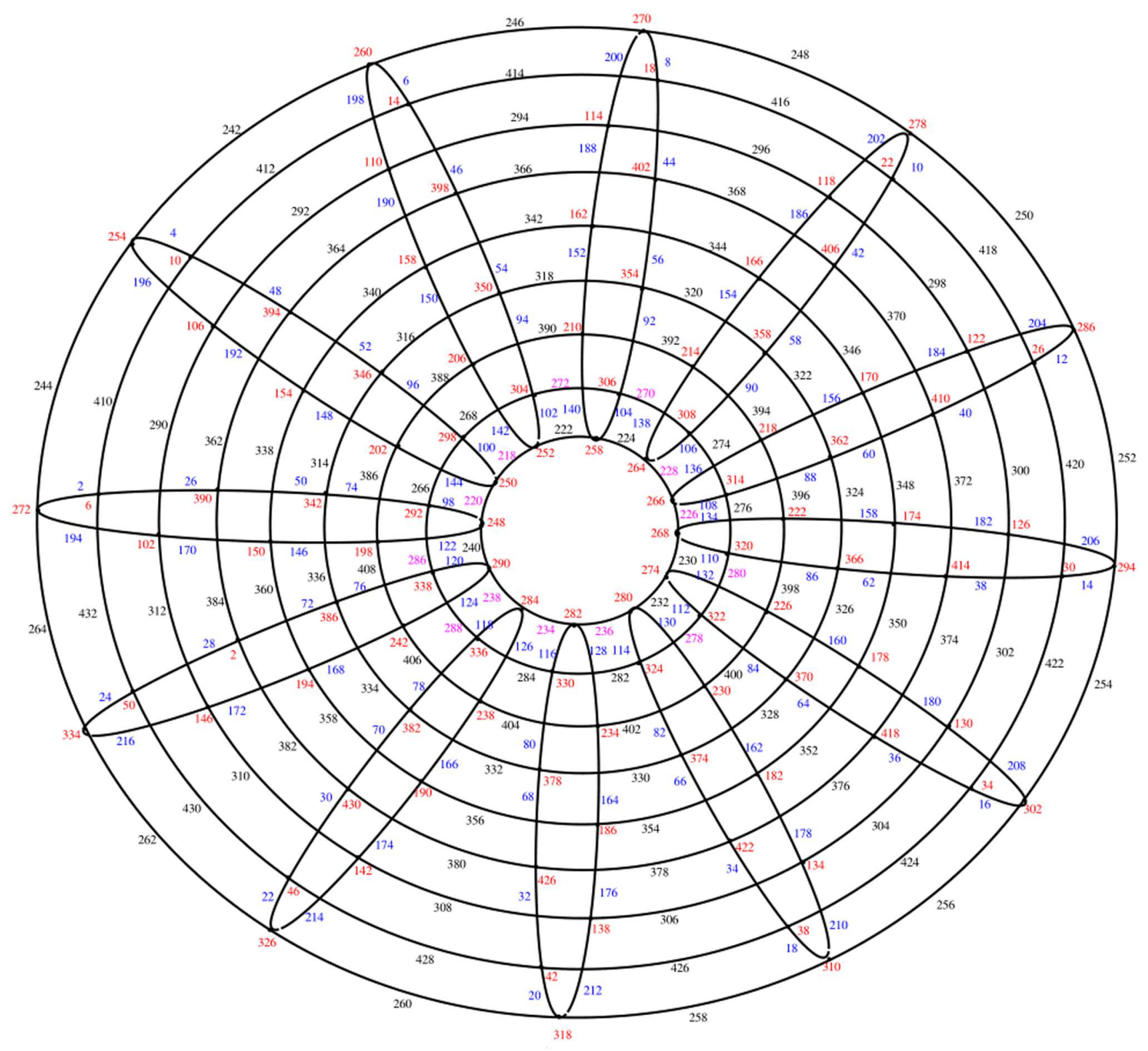

(c) $T_{12,9}$ 


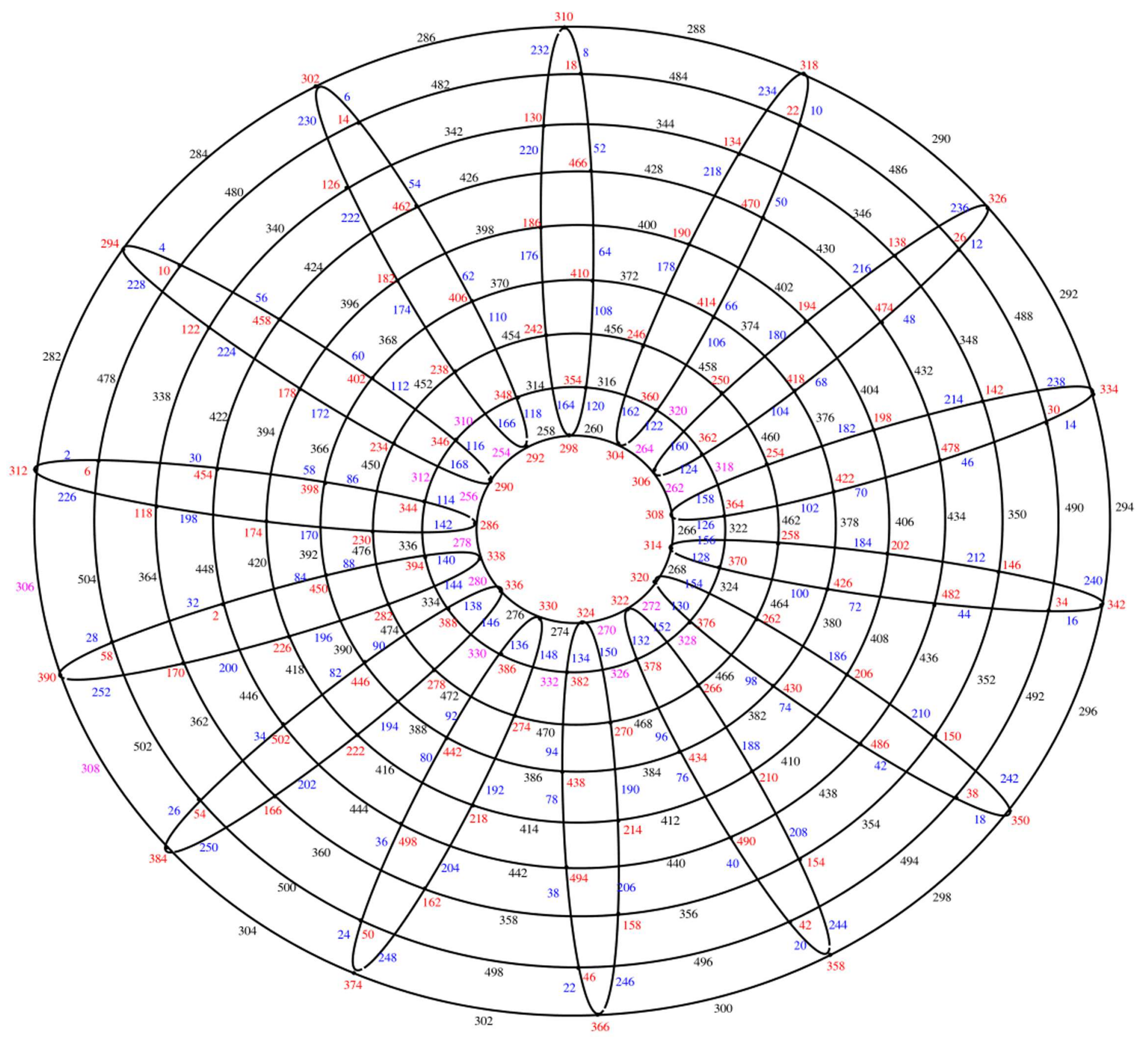

(d) $T_{14,9}$

Figure 2.13: The e. e. g. 1. of $T_{8,9}, T_{10,9}, T_{12,9}$ and $T_{14,9}$ 
Theorem 2.0.7. Assume that $p>1$ is an odd number that belongs to $\mathbf{Z}^{+}$ and $q>1$ is an odd number that belongs to $\mathbf{Z}^{+}$. Then the torus grid graph $T_{p, q}$ is an e. e. g. graph.

Proof. By taking $n=\left|V\left(T_{p, q}\right)\right|=p q$ and $m=\left|E\left(T_{p, q}\right)\right|=2 p q$ and $r=\max \{m, n\}=2 p q$ and let $g: E\left(T_{p, q}\right) \longrightarrow\{2,4, \cdots, 4 p q-2\}$, then there are four cases:

Case $1 q \equiv 0 \bmod 8$. Assume that $T_{p, q}$, is given as in Figure2.14. Here, we label the edges of the ovals $C_{p}^{(j)}, 1 \leq j \leq q$ starting with the edges of the oval $C_{p}^{(1)}$ as follows :

Turn clockwise to label the edges

$$
\begin{gathered}
u_{(p-1) q+1} u_{(p-2) q+1}, u_{(p-1) q+2} u_{(p-2) q+2}, u_{(p-1) q+3} u_{(p-2) q+3} \\
\cdots, u_{p q-1} u_{(p-1) q-1}, u_{p q} u_{(p-1) q}
\end{gathered}
$$

by $2,4,6, \cdots, 2 q-2,2 q$, then turn counter-clockewise to label the edges

$$
\begin{aligned}
& u_{(p-2) q+1} u_{(p-4) q+1}, u_{(p-1) q} u_{(p-3) q}, u_{(p-1) q-1} u_{(p-3)-q-1}, \\
& \quad \cdots, u_{(p-2) q+3} u_{(p-4) q+3}, u_{(p-2) q+2} u_{(p-4) q+2}
\end{aligned}
$$

by $2 q+2,2 q+4,2 q+6, \cdots, 4 q-2,4 q$ and so on. If $p \equiv 1 \bmod 4$, we turn clockwise to label the edges

$$
u_{q+1} u_{1}, u_{q+2} u_{2}, u_{q+3} u_{3}, \cdots, u_{2 q-1} u_{q-1}, u_{2 q} u_{q}
$$

by $(p-1) q+2,(p-1) q+4,(p-1) q+6, \cdots,(p+1) q-2,(p+1) q$, next we turn-counterclockwise to label the edges

$$
u_{1} u_{2 q+1}, u_{q} u_{3 q}, u_{q-1} u_{3 q-1}, \cdots, u_{3} u_{2 q+3}, u_{2} u_{2 q+2}
$$

by $(p+1) q+2,(p+1) q+4,(p+1) q+6, \cdots,(p+3) q-2,(p+3) q$, then we turn clockwise to label the edges

$$
u_{2 q+1} u_{4 q+1}, u_{2 q+2} u_{4 q+2}, u_{2 q+3} u_{4 q+3}, \cdots, u_{3 q-1} u_{5 q-1}, u_{3 q} u_{5 q}
$$

by $(p+3) q+2,(p+3) q+4,(p+3) q+6, \cdots,(p+5) q-2,(p+5) q$ and so on. 


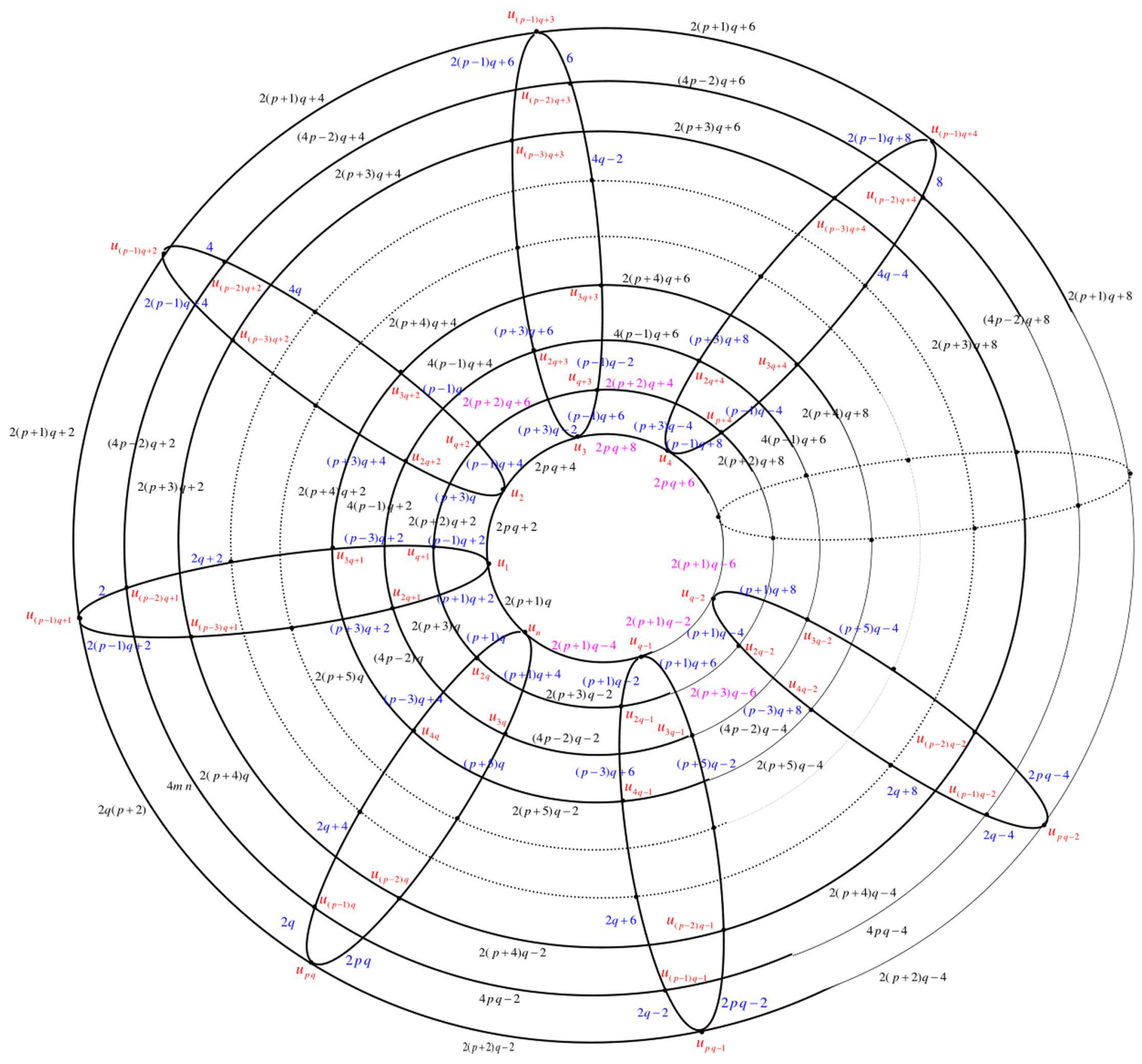

Figure 2.14: $T_{p, q}, p$ is even and $q \equiv 0 \bmod 8$ 
If $p \equiv 3 \bmod 4$,we turn counterm-clockwise to label the edges

$$
u_{q+1} u_{1}, u_{2 q} u_{q}, u_{2 q-1} u_{q-1}, \cdots, u_{q+3} u_{3}, u_{q+2} u_{2}
$$

by $(p-1) q+2,(p-1) q+4,(p-1) q+6, \cdots,(p+1) q-2,(p+1) q$, next we turn clockwise to label the edges

$$
u_{1} u_{2 q+1}, u_{2} u_{2 q+2}, u_{3} u_{2 q+3}, \cdots, u_{q-1} u_{3 q-1}, u_{q} u_{3 q}
$$

by $(p+1) q+2,(p+1) q+4,(p+1) q+6, \cdots,(p+3) q-2,(p+3) q$, then turn counter-clockwise anticlockwise to label the edges

$$
u_{2 q+1} u_{4 q+1}, u_{3 q} u_{5 q}, u_{3 q-1} u_{5 q-1}, \cdots, u_{2 q+3} u_{4 q+3}, u_{2 q+2} u_{4 q+2}
$$

by $(p+3) q+2,(p+3) q+4,(p+3) q+6, \cdots,(p+5) q-2,(p+5) q,(p+5) q-$ $2,(p+5) q$ and so on.

Finally, we turn clockewise to label the edges

$$
\begin{gathered}
u_{(p-3) q+1} u_{(p-1) q+1}, u_{(p-3) q+2} u_{(p-1) q+2}, u_{(p-3) q+3} u_{(p-1) q+3}, \\
\cdots, u_{(p-2) q-1} u_{p q-1}, u_{(p-2) q} u_{p q}
\end{gathered}
$$

by $2 q(p-1)+2,2 q(p-1)+4,2 q(p-1)+6, \cdots, 2 p q-2,2 p q$. Second, we label the edges of the circles $C_{q}^{(j)}, 1 \leq j \leq p$ starting with the edges of the inner most circle $C_{q}^{(1)}$ as follows:

$g\left(u_{1} u_{2}\right)=2 p q+2, g\left(u_{2} u_{3}\right)=2 p q+4, g\left(u_{3} u_{4}\right)=2 p q+8, g\left(u_{4} u_{5}\right)=2 p q+$ $6, g\left(u_{5} u_{6}\right)=2 p q+10, g\left(u_{6} u_{7}\right)=2 p q+12, g\left(u_{7} u_{8}\right)=2 p q+16, g\left(u_{8} u_{9}\right)=$ $2 p q+14, g\left(u_{9} u_{10}\right)=2 p q+18, g\left(u_{10} u_{11}\right)=2 p q+20, g\left(u_{11} u_{12}\right)=2 p q+$ $24, \cdots, g\left(u_{q-9} u_{q-8}\right)=2 q(p+1)-20, g\left(u_{q-8} u_{q-7}\right)=2 q(p+1)-16, g\left(u_{q-7} u_{q-6}\right)=$ $2 q(p+1)-14, g\left(u_{q-6} u_{q-5}\right)=2 q(p+1)-10, g\left(u_{q-5} u_{q-4}\right)=2 q(p+1)-$ $12, g\left(u_{q-4} u_{q-3}\right)=2 q(p+1)-8, g\left(u_{q-3} u_{q-2}\right)=2 q(p+1)-6, g\left(u_{q-2} u_{q-1}\right)=$ $2 q(p+1)-2, g\left(u_{q-1} u_{q}\right)=2 q(p+1)-4$ and $g\left(u_{q} u_{1}\right)=2 q(p+1)$, next we lable the edges of outer circle $C_{q}^{(p)}$ as follows: $g\left(u_{(p-1) q+i} u_{(p-1) q+i-1}\right)=$ $2 q(p+1)+2 i, 1 \leq i \leq q-1, g\left(u_{p q} u_{q(p-1)+1}\right)=2 q(p+2)$, then we label the edges the circle $C_{q}^{(2)}$ as : $g\left(u_{q+1} u_{q+2}\right)=2 q(p+2)+2, g\left(u_{q+2} u_{q+3}\right)=$ $2 q(p+2)+6, g\left(u_{q+3} u_{q+4}\right)=2 q(p+2)+4, g\left(u_{q+4} u_{q+5}\right)=2 q(p+2)+$ $8, g\left(u_{q+5} u_{q+6}\right)=2 q(p+2)+10, g\left(u_{q+6} u_{q+7}\right)=2 q(p+2)+14, g\left(u_{q+7} u_{q+8}\right)=$ $2 q(p+2)+12, g\left(u_{q+8} u_{q+9}\right)=2 q(p+2)+16, g\left(u_{q+9} u_{q+10}\right)=2 q(p+2)+$ $18, g\left(u_{q+10} u_{q+11}\right)=2 q(p+2)+22, \cdots, g\left(u_{2 q+9} u_{2 q+8}\right)=2 q(p+3)-16, g\left(u_{2 q+8} u_{2 q+7}\right)=$ $2 q(p+3)-12, g\left(u_{2 q+7} u_{2 q+6}\right)=2 q(p+3)-14, g\left(u_{2 q+6} u_{2 q+5}\right)=2 q(p+3)-$ 
$10, g\left(u_{2 q+5} u_{2 q+4}\right)=2 q(p+3)-8, g\left(u_{2 q+4} u_{2 q+3}\right)=2 q(p+3)-4, g\left(u_{2 q+3} u_{2 q+2}\right)=$ $2 q(p+3)-6, g\left(u_{2 q+2} u_{2 q+1}\right)=2 q(p+3)-2, g\left(u_{2 q-1} u_{2 q}\right)=2 q(p+3)-2$ and $g\left(u_{2 q} u_{q+1}\right)=2 q(p+3)$. Then we label the edges of circles $C_{q}^{(3)}, C_{q}^{(5)}, \cdots, C_{q}^{(p-2)}$ as follows:

$g\left(u_{(j-1) q+i} u_{(j-1) q+i+1}\right)=4 q\left(p-\frac{j-1}{2}\right)+2 i$ and $g\left(u_{j q} u_{(j-1) q+1}\right)=4 q(p-$ $(2 j-4))-2,3 \leq j \leq p-2$ where $j$ is odd and $1 \leq i \leq q-1$. Finally, we label the edges of of the circles $C_{q}^{(4)}, C_{q}^{(6)}, \cdots, C_{q}^{(p-1)}$ as follows:

$g\left(u_{(j-1) q+i} u_{(j-1) q+i+1}\right)=2 q(p+j)+2 i$ and $g\left(u_{j q} u_{(j-1) q+1}\right)=2 q(p+j+1)$, $4 \leq j \leq q-1$ where $j$ is even and $1 \leq i \leq q-1$. Thus we have the labels of the vertices of the inner most circle $C_{q}^{(1)} \bmod 4 p q$ are $g^{*}\left(u_{1}\right) \equiv$ $2 p q+2 q+6, g^{*}\left(u_{2}\right) \equiv 2 p q+2 q+10, g^{*}\left(u_{3}\right) \equiv 2 p q+2 q+16, g^{*}\left(u_{4}\right) \equiv$ $2 p q+2 q+18, g^{*}\left(u_{5}\right) \equiv 2 p q+2 q+20, g^{*}\left(u_{6}\right) \equiv 2 p q+2 q+26, g^{*}\left(u_{7}\right) \equiv$ $2 p q+2 q+32, g^{*}\left(u_{8}\right) \equiv 2 p q+2 q+34, g^{*}\left(u_{9}\right) \equiv 2 p q+2 q+36, g^{*}\left(u_{10}\right) \equiv$ $2 p q+2 q+42, g^{*}\left(u_{11}\right) \equiv 2 p q+2 q+48, g^{*}\left(u_{12}\right) \equiv 2 p q+2 q+50, \cdots, g^{*}\left(u_{q-8}\right) \equiv$ $2 p q+6 q-32, g^{*}\left(u_{q-7}\right) \equiv 2 p q+6 q-26, g^{*}\left(u_{q-6}\right) \equiv 2 p q+6 q-20, g^{*}\left(u_{q-5}\right) \equiv$ $2 p q+6 q-18, g^{*}\left(u_{q-4}\right) \equiv 2 p q+6 q-16, g^{*}\left(u_{q-3}\right) \equiv 2 p q+6 q-10, g^{*}\left(u_{q-2}\right) \equiv$ $2 p q+6 q-4, g^{*}\left(u_{q-1}\right) \equiv 2 p q+6 q-2$ and $g^{*}\left(u_{q}\right) \equiv 2 p q+6 q$. The labels of the vertices of the outer circle $C_{q}^{(p)} \bmod 4 p q$ are:

$g^{*}\left(u_{(p-1) q+1}\right) \equiv 2 p q+4 q+6, g^{*}\left(u_{(p-1) q+i}\right) \equiv 2 p q+2 q+8 i-2,2 \leq i \leq q$ The labels of the vertices of the outer circle $C_{q}^{(2)} \bmod 4 p q$ are: $g^{*}\left(u_{q+1}\right) \equiv$ $2 p q+6 q+6, g^{*}\left(u_{q+2}\right) \equiv 2 p q+6 q+12, g^{*}\left(u_{q+3}\right) \equiv 2 p q+6 q+14, g^{*}\left(u_{q+4}\right) \equiv$ $2 p q+6 q+18, g^{*}\left(u_{q+5}\right) \equiv 2 p q+6 q+22, g^{*}\left(u_{q+6}\right) \equiv 2 p q+6 q+28, g^{*}\left(u_{q+7}\right) \equiv$ $2 p q+6 q+30, g^{*}\left(u_{q+8}\right) \equiv 2 p q+6 q+32, g^{*}\left(u_{q+9}\right) \equiv 2 p q+6 q+38, g^{*}\left(u_{q+10}\right) \equiv$ $2 p q+6 q+44 \cdots, g^{*}\left(u_{2 q-8}\right) \equiv 2 p q+10 q-30, g^{*}\left(u_{2 q-7}\right) \equiv 2 p q+10 q-$ $24, g^{*}\left(u_{2 q-6}\right) \equiv 2 p q+10 q-22, g^{*}\left(u_{2 q-5}\right) \equiv 2 p q+10 q-20, g^{*}\left(u_{2 q-4}\right) \equiv 2 p q+$ $10 q-14, g^{*}\left(u_{2 q-3}\right) \equiv 2 p q+10 q-8, g^{*}\left(u_{2 q-2}\right) \equiv 2 p q+10 q-6, g^{*}\left(u_{2 q-1}\right) \equiv$ $2 p q+10 q-4$ and $g^{*}\left(u_{2 q}\right) \equiv 2 p q+10 q+2$. The labels of the vertices of circles $C_{q}^{(3)}, C_{q}^{(5)}, \cdots, C_{q}^{(p-2)} \bmod 4 p q$ are: $g^{*}\left(u_{j q+i}\right) \equiv 2 p q-2(j-1) q+4 i+2,2 \leq$ $j \leq p-3$ where $j$ is even and $1 \leq i \leq p$. 
Case $2 p \equiv 3 \bmod 8$. Let $T_{p, q}$ be as in Figure2.14. First, we label the edges of the ovals $C_{p}^{(j)}, 1 \leq j \leq n$ as the same in case (1). Secondly, we label the edges of the circles $C_{q}^{(j)}, 1 \leq j \leq p$ begin with the edges of the inner most circle $C_{q}^{(1)}$ as follows: $g\left(u_{1} u_{2}\right)=2 p q+2, g\left(u_{2} u_{3}\right)=2 p q+4, g\left(u_{3} u_{4}\right)=$ $2 p q+8, g\left(u_{4} u_{5}\right)=2 p q+6, g\left(u_{5} u_{6}\right)=2 p q+10, g\left(u_{6} u_{7}\right)=2 p q+12, g\left(u_{7} u_{8}\right)=$ $2 p q+16, g\left(u_{8} u_{9}\right)=2 p q+14, g\left(u_{9} u_{10}\right)=2 p q+18, g\left(u_{10} u_{11}\right)=2 p q+$ $20, g\left(u_{11} u_{12}\right)=2 p q+24, \cdots, g\left(u_{q-9} u_{q-8}\right)=2 q(p+1)-18, g\left(u_{q-8} u_{q-7}\right)=$ $2 q(p+1)-14, g\left(u_{q-7} u_{q-6}\right)=2 q(p+1)-16, g\left(u_{q-6} u_{q-5}\right)=2 q(p+1)-$ $12, g\left(u_{q-5} u_{q-4}\right)=2 q(p+1)-10, g\left(u_{q-4} u_{q-3}\right)=2 q(p+1)-6, g\left(u_{q-3} u_{q-2}\right)=$ $2 q(p+1)-8, g\left(u_{q-2} u_{q-1}\right)=2 q(p+1), g\left(u_{q-1} u_{q}\right)=2 q(p+1)-7$ and $g\left(u_{q} u_{1}\right)=2 q(p+1)-2$. Then we label the edges of circle $C_{q}^{(2)}$ as follows: $g\left(u_{q+1} u_{q+2}\right)=2 q(p+2)+2, g\left(u_{q+2} u_{q+3}\right)=2 q(p+2)+6, g\left(u_{q+3} u_{q+4}\right)=$ $2 q(p+2)+4, g\left(u_{q+4} u_{q+5}\right)=2 q(p+2)+8, g\left(u_{q+5} u_{q+6}\right)=2 q(p+2)+$ $10, g\left(u_{q+6} u_{q+7}\right)=2 q(p+2)+14, g\left(u_{q+7} u_{q+8}\right)=2 q(p+2)+12, g\left(u_{q+8} u_{q+9}\right)=$ $2 q(p+2)+16, g\left(u_{q+9} u_{q+10}\right)=2 q(p+2)+18, g\left(u_{q+10} u_{q+11}\right)=2 q(p+$ $2)+22, \cdots, g\left(u_{2 q-9} u_{2 q-8}\right)=2 q(p+3)-16, g\left(u_{2 q-8} u_{2 q-7}\right)=2 q(p+3)-$ $18, g\left(u_{2 q-7} u_{2 q-6}\right)=2 q(p+3)-14, g\left(u_{2 q-6} u_{2 q-5}\right)=2 q(p+3)-12, g\left(u_{2 q-5} u_{2 q-4}\right)=$ $2 q(p+3)-8, g\left(u_{2 q-4} u_{2 q-3}\right)=2 q(p+3)-10, g\left(u_{2 q-3} u_{2 q-2}\right)=2 q(p+3)-$ $6, g\left(u_{2 q-2} u_{2 q-1}\right)=2 q(p+3)-4, g\left(u_{2 q-1} u_{2 q}\right)=2 q(p+3)$ and $g\left(u_{2 q} u_{q+1}\right)=$ $2 q(p+3)-2$. Then we label the edges of circles $C_{q}^{(3)}, C_{q}^{(4)}, \cdots, C_{q}^{(p)}$ as the same iq case (1). Thus, we have the labels of the vertices of the inner most circle $C_{q}^{(1)} \bmod 4 p q$ are: $g^{*}\left(u_{1}\right) \equiv 2 p q+2 q+4, g^{*}\left(u_{2}\right) \equiv 2 p q+2 q+10, g^{*}\left(u_{3}\right) \equiv$ $2 p q+2 q+16, g^{*}\left(u_{4}\right) \equiv 2 p q+2 q+18, g^{*}\left(u_{5}\right) \equiv 2 p q+2 q+20, g^{*}\left(u_{6}\right) \equiv$ $2 p q+2 q+26, g^{*}\left(u_{7}\right) \equiv 2 p q+2 q+32, g^{*}\left(u_{8}\right) \equiv 2 p q+2 q+34, g^{*}\left(u_{9}\right) \equiv$ $2 p q+2 q+36, g^{*}\left(u_{10}\right) \equiv 2 p q+2 q+42, g^{*}\left(u_{11}\right) \equiv 2 p q+2 q+48, g^{*}\left(u_{12}\right) \equiv 2 p q+$ $2 q+50, \cdots, g^{*}\left(u_{q-8}\right) \equiv 2 p q+6 q-28, g^{*}\left(u_{q-7}\right) \equiv 2 p q+6 q-26, g^{*}\left(u_{q-6}\right) \equiv$ $2 p q+6 q-24, g^{*}\left(u_{q-5}\right) \equiv 2 p q+6 q-18, g^{*}\left(u_{q-4}\right) \equiv 2 p q+6 q-12, g^{*}\left(u_{q-3}\right) \equiv$ $2 p q+6 q-10, g^{*}\left(u_{q-2}\right) \equiv 2 p q+6 q-4, g^{*}\left(u_{q-1}\right) \equiv 2 p q+6 q$ and $g^{*}\left(u_{q}\right) \equiv$ $2 p q+6 q-2$. The labels of the vertices of the circle $C_{q}^{(2)} \bmod 4 p q$ are as follows: $g^{*}\left(u_{q+1}\right) \equiv 2 p q+6 q+4, g^{*}\left(u_{q+2}\right) \equiv 2 p q+6 q+12, g^{*}\left(u_{q+3}\right) \equiv$ $2 p q+6 q+14, g^{*}\left(u_{q+4}\right) \equiv 2 p q+6 q+16, g^{*}\left(u_{q+5}\right) \equiv 2 p q+6 q+22, g^{*}\left(u_{q+6}\right) \equiv$ $2 p q+6 q+28, g^{*}\left(u_{q+7}\right) \equiv 2 p q+6 q+30, g^{*}\left(u_{q+8}\right) \equiv 2 p q+6 q+32, g^{*}\left(u_{q+9}\right) \equiv$ $2 p q+6 q+38, g^{*}\left(u_{q+10}\right) \equiv 2 p q+6 q+44 \cdots, g^{*}\left(u_{2 q-8}\right) \equiv 2 p q+10 q-$ $30, g^{*}\left(u_{2 q-7}\right) \equiv 2 p q+10 q-28, g^{*}\left(u_{2 q-6}\right) \equiv 2 p q+10 q-22, g^{*}\left(u_{2 q-5}\right) \equiv 2 p q+$ $10 q-16, g^{*}\left(u_{2 q-4}\right) \equiv 2 p q+10 q-14, g^{*}\left(u_{2 q-3}\right) \equiv 2 p q+10 q-12, g^{*}\left(u_{2 q-2}\right) \equiv$ $2 p q+10 q-6, g^{*}\left(u_{2 q-1}\right) \equiv 2 p q+10 q$ and $g^{*}\left(u_{2 p}\right) \equiv 2 p q+10 q+2$. The labels of the vertices of circles $C_{q}^{(3)}, C_{q}^{(4)}, \cdots, C_{q}^{(p)} \bmod 4 p q$ are the same as in case (1). 
Case $3 p \equiv 5 \bmod 8$. Let $T_{p, q}$ be as in Figure14. First, label the edges of the ovals $C_{p}^{(j)}, 1 \leq j \leq q$ as the same in case (1). Secondly, we label the edges of the circles $C_{q}^{(j)}, 1 \leq j \leq p$ begin with the edges of the inner most circle $C_{q}^{(1)}$ as follows: $g\left(u_{1} u_{2}\right)=2 p q+4, g\left(u_{2} u_{3}\right)=2 p q+2, g\left(u_{3} u_{4}\right)=$ $2 p q+6, g\left(u_{4} u_{5}\right)=2 p q+8, g\left(u_{5} u_{6}\right)=2 p q+12, g\left(u_{6} u_{7}\right)=2 p q+10, g\left(u_{7} u_{8}\right)=$ $2 p q+14, g\left(u_{8} u_{9}\right)=2 p q+16, g\left(u_{9} u_{10}\right)=2 p q+20, g\left(u_{10} u_{11}\right)=2 p q+$ $18, g\left(u_{11} u_{12}\right)=2 p q+22, \cdots, g\left(u_{q-9} u_{q-8}\right)=2 q(p+1)-18, g\left(u_{q-8} u_{q-7}\right)=$ $2 q(p+1)-14, g\left(u_{q-7} u_{q-6}\right)=2 q(p+1)-16, g\left(u_{q-6} u_{q-5}\right)=2 q(p+1)-$ $12, g\left(u_{q-5} u_{q-4}\right)=2 q(p+1)-10, g\left(u_{q-4} u_{q-3}\right)=2 q(p+1)-6, g\left(u_{q-3} u_{q-2}\right)=$ $2 q(p+1)-8, g\left(u_{q-2} u_{q-1}\right)=2 q(p+1), g\left(u_{q-1} u_{q}\right)=2 q(p+1)-4 a q d g\left(u_{q} u_{1}\right)=$ $2 q(p+1) g\left(v_{(m-1) n+i} v_{(m-1) n+i+1}\right)=2 n(m+1)+2 i, 3 \leq i \leq n-2$ and $g\left(v_{m n} v_{n(m-1)+1}\right)=2 n(m+2)$. Next we label the edges the circle $C_{q}^{(2)}$ as follows: $g\left(u_{q+1} u_{q+2}\right)=2 q(p+2)+2, g\left(u_{q+2} u_{q+3}\right)=2 q(p+2)+6, g\left(u_{q+3} u_{q+4}\right)=$ $2 q(p+2)+4, g\left(u_{q+4} u_{q+5}\right)=2 q(p+2)+8, g\left(u_{q+5} u_{q+6}\right)=2 q(p+2)+$ $10, g\left(u_{q+6} u_{q+7}\right)=2 q(p+2)+14, g\left(u_{q+7} u_{q+8}\right)=2 q(p+2)+12, g\left(u_{q+8} u_{q+9}\right)=$ $2 q(p+2)+16, g\left(u_{q+9} u_{q+10}\right)=2 q(p+2)+18, g\left(u_{q+10} u_{q+11}\right)=2 q(p+$ $2)+22, \cdots, g\left(u_{2 q-9} u_{2 q-8}\right)=2 q(p+3)-16, g\left(u_{2 q-8} u_{2 q-7}\right)=2 q(p+3)-$ $12, g\left(u_{2 q-7} u_{2 q-6}\right)=2 q(p+3)-14, g\left(u_{2 q-6} u_{2 q-5}\right)=2 q(p+3)-10, g\left(u_{2 q-5} u_{2 q-4}\right)=$ $2 q(p+3)-8, g\left(u_{2 q-4} u_{2 q-3}\right)=2 q(p+3)-4, g\left(u_{2 q-3} u_{2 q-2}\right)=2 q(p+$ $3)-4, g\left(u_{2 q-2} u_{2 q-1}\right)=2 q(p+3)-6, g\left(u_{2 q-1} u_{2 q}\right)=2 q(p+3)-2$ and $g\left(u_{2 q} u_{q+1}\right)=2 q(p+3)$.

Then we label the edges of circles $C_{q}^{(3)}, C_{q}^{(4)}, \cdots, C_{q}^{(p)}$ and as the same in case (1).

Thus, the labels of the vertices of the inner most circle $C_{q}^{(1)} \bmod 4 p q$ are: $g^{*}\left(u_{1}\right) \equiv 2 p q+2 q+6, g^{*}\left(u_{2}\right) \equiv 2 p q+2 q+10, g^{*}\left(u_{3}\right) \equiv 2 p q+2 q+12, g^{*}\left(u_{4}\right) \equiv$ $2 p q+2 q+18, g^{*}\left(u_{5}\right) \equiv 2 p q+2 q+24, g^{*}\left(u_{6}\right) \equiv 2 p q+2 q+26, g^{*}\left(u_{7}\right) \equiv$ $2 p q+2 q+28, g^{*}\left(u_{8}\right) \equiv 2 p q+2 q+34, g^{*}\left(u_{9}\right) \equiv 2 p q+2 q+40, g^{*}\left(u_{10}\right) \equiv$ $2 p q+2 q+42, g^{*}\left(u_{11}\right) \equiv 2 p q+2 q+44, g^{*}\left(u_{12}\right) \equiv 2 p q+2 q+50, \cdots, g^{*}\left(u_{q-8}\right) \equiv$ $2 p q+6 q-28, g^{*}\left(u_{q-7}\right) \equiv 2 p q+6 q-26, g^{*}\left(u_{q-6}\right) \equiv 2 p q+6 q-24, g^{*}\left(u_{q-5}\right) \equiv$ $2 p q+6 q-18, g^{*}\left(u_{q-4}\right) \equiv 2 p q+6 q-12, g^{*}\left(u_{q-3}\right) \equiv 2 p q+6 q-10, g^{*}\left(u_{q-2}\right) \equiv$ $2 p q+6 q-4, g^{*}\left(u_{q-1}\right) \equiv 2 p q+6 q$ and $g^{*}\left(u_{q}\right) \equiv 2 p q+6 q-2$. The labels of the vertices of the outer circle $C_{q}^{(2)} \bmod 4 p q$ are as follows:

$g^{*}\left(u_{q+1}\right) \equiv 2 p q+6 q+6, g^{*}\left(u_{q+2}\right) \equiv 2 p q+6 q+12, g^{*}\left(u_{q+3}\right) \equiv 2 p q+$ $6 q+14, g^{*}\left(u_{q+4}\right) \equiv 2 p q+6 q+16, g^{*}\left(u_{q+5}\right) \equiv 2 p q+6 q+22, g^{*}\left(u_{q+6}\right) \equiv$ $2 p q+6 q+28, g^{*}\left(u_{q+7}\right) \equiv 2 p q+6 q+30, g^{*}\left(u_{q+8}\right) \equiv 2 p q+6 q+32, g^{*}\left(u_{q+9}\right) \equiv$ $2 p q+6 q+38, g^{*}\left(u_{q+10}\right) \equiv 2 p q+6 q+44, \cdots, g^{*}\left(u_{2 q-8}\right) \equiv 2 p q+10 q-$ $30, g^{*}\left(u_{2 q-7}\right) \equiv 2 p q+10 q-24, g^{*}\left(u_{2 q-6}\right) \equiv 2 p q+10 q-22, g^{*}\left(u_{2 q-5}\right) \equiv 2 p q+$ $10 q-20, g^{*}\left(u_{2 q-4}\right) \equiv 2 p q+10 q-14, g^{*}\left(u_{2 q-3}\right) \equiv 2 p q+10 q-8, g^{*}\left(u_{2 q-2}\right) \equiv$ 
$2 p q+10 q-6, g^{*}\left(u_{2 q-1}\right) \equiv 2 p q+10 q-4, g^{*}\left(u_{2 q}\right) \equiv 2 p q+10 q+2$. Then we label the edges of circles $C_{q}^{(3)}, C_{q}^{(4)}, \cdots, C_{q}^{(p)}$ and $\bmod 4 p q$ as the same in case (1).

Case $4 p \equiv 7 \bmod 8$. Let $T_{p, q}$ be as in Figure 2.14. First, we label the edges of the ovals $C_{p}^{(j)}, 1 \leq j \leq q$ as the same in case (1). Secondly, we label the edges of the circles $C_{q}^{(j)}, 1 \leq j \leq p$ begin with the edges of the inner most circle $C_{q}^{(1)}$ as follows: $g\left(u_{1} u_{2}\right)=2 p q+4, g\left(u_{2} u_{3}\right)=2 p q+2, g\left(u_{3} u_{4}\right)=$ $2 p q+6, g\left(u_{4} u_{5}\right)=2 p q+8, g\left(u_{5} u_{6}\right)=2 p q+12, g\left(u_{6} u_{7}\right)=2 p q+10, g\left(u_{7} u_{8}\right)=$ $2 p q+14, g\left(u_{8} u_{9}\right)=2 p q+16, g\left(u_{9} u_{10}\right)=2 p q+20, g\left(u_{10} u_{11}\right)=2 p q+$ $18, g\left(u_{11} u_{12}\right)=2 p q+22, \cdots, g\left(u_{q-9} u_{q-8}\right)=2 q(p+1)-20, g\left(u_{q-8} u_{q-7}\right)=$ $2 q(p+1)-16, g\left(u_{q-7} u_{q-6}\right)=2 q(p+1)-14, g\left(u_{q-6} u_{q-5}\right)=2 q(p+1)-$ $10, g\left(u_{q-5} u_{q-4}\right)=2 q(p+1)-12, g\left(u_{q-4} u_{q-3}\right)=2 q(p+1)-8, g\left(u_{q-3} u_{q-2}\right)=$ $2 q(p+1)-6, g\left(u_{q-2} u_{q-1}\right)=2 q(p+1)-2, g\left(u_{q-1} u_{q}\right)=2 q(p+1)-4$ and $g\left(u_{q} u_{1}\right)=2 q(p+1)$. Next, we label the edges the circle $C_{q}^{(2)}$ as follows: $g\left(u_{q+1} u_{q+2}\right)=2 q(p+2)+2, g\left(u_{q+2} u_{q+3}\right)=2 q(p+2)+6, g\left(u_{q+3} u_{q+4}\right)=$ $2 q(p+2)+4, g\left(u_{q+4} u_{q+5}\right)=2 q(p+2)+8, g\left(u_{q+5} u_{q+6}\right)=2 q(p+2)+$ $10, g\left(u_{q+6} u_{q+7}\right)=2 q(p+2)+14, g\left(u_{q+7} u_{q+8}\right)=2 q(p+2)+12, g\left(u_{q+8} u_{q+9}\right)=$ $2 q(p+2)+16, g\left(u_{q+9} u_{q+10}\right)=2 q(p+2)+18, g\left(u_{q+10} u_{q+11}\right)=2 q(p+$ $2)+22, \cdots, g\left(u_{2 q-9} u_{2 q-8}\right)=2 q(p+3)-16, g\left(u_{2 q-8} u_{2 q-7}\right)=2 q(p+3)-$ $18, g\left(u_{2 q-7} u_{2 q-6}\right)=2 q(p+3)-14, g\left(u_{2 q-6} u_{2 q-5}\right)=2 q(p+3)-12, g\left(u_{2 q-5} u_{2 q-4}\right)=$ $2 q(p+3)-8, g\left(u_{2 q-4} u_{2 q-3}\right)=2 q(p+3)-10, g\left(u_{2 q-3} u_{2 q-2}\right)=2 q(p+3)-$ $6, g\left(u_{2 q-2} u_{2 q-1}\right)=2 q(p+3)-4, g\left(u_{2 q-1} u_{2 q}\right)=2 q(p+3)$ and $g\left(u_{2 q} u_{q+1}\right)=$ $2 q(p+3)-2$. Hence, we label the edges of circles $C_{q}^{(3)}, C_{q}^{(4)}, \cdots, C_{q}^{(p)}$ as the same in case (1).

Thus, the labels of the vertices of the inner most circle $C_{q}^{(1)} \bmod 4 p q$ are: $g^{*}\left(u_{1}\right) \equiv 2 p q+2 q+8, g^{*}\left(u_{2}\right) \equiv 2 p q+2 q+10, g^{*}\left(u_{3}\right) \equiv 2 p q+2 q+12, g^{*}\left(u_{4}\right) \equiv$ $2 p q+2 q+18, g^{*}\left(u_{5}\right) \equiv 2 p q+2 q+24, g^{*}\left(u_{6}\right) \equiv 2 p q+2 q+26, g^{*}\left(u_{7}\right) \equiv$ $2 p q+2 q+28, g^{*}\left(u_{8}\right) \equiv 2 p q+2 q+34, g^{*}\left(u_{9}\right) \equiv 2 p q+2 q+40, g^{*}\left(u_{10}\right) \equiv$ $2 p q+2 q+42, g^{*}\left(u_{11}\right) \equiv 2 p q+2 q+44, g^{*}\left(u_{12}\right) \equiv 2 p q+2 q+50, \cdots, g^{*}\left(u_{q-8}\right) \equiv$ $2 p q+6 q-32, g^{*}\left(u_{q-7}\right) \equiv 2 p q+6 q-26, g^{*}\left(u_{q-6}\right) \equiv 2 p q+6 q-20, g^{*}\left(u_{q-5}\right) \equiv$ $2 p q+6 q-18, g^{*}\left(u_{q-4}\right) \equiv 2 p q+6 q-16, g^{*}\left(u_{q-3}\right) \equiv 2 p q+6 q-10, g^{*}\left(u_{q-2}\right) \equiv$ $2 p q+6 q-4, g^{*}\left(u_{q-1}\right) \equiv 2 p q+6 q-2$ and $g^{*}\left(u_{q}\right) \equiv 2 p q+6 q$.

The labels of the vertices of the circle $C_{q}^{(2)} \bmod 4 p q$ are: $g^{*}\left(u_{q+1}\right) \equiv 2 p q+6 q+4, g^{*}\left(u_{q+2}\right) \equiv 2 p q+6 q+12, g^{*}\left(u_{q+3}\right) \equiv 2 p q+$ $6 q+14, g^{*}\left(u_{q+4}\right) \equiv 2 p q+6 q+16, g^{*}\left(u_{q+5}\right) \equiv 2 p q+6 q+22, g^{*}\left(u_{q+6}\right) \equiv$ $2 p q+6 q+28, g^{*}\left(u_{q+7}\right) \equiv 2 p q+6 q+30, g^{*}\left(u_{q+8}\right) \equiv 2 p q+6 q+32, g^{*}\left(u_{q+9}\right) \equiv$ 
circle $C_{q}^{(2)} \bmod 4 p q$ are:

$g^{*}\left(u_{q+1}\right) \equiv 2 p q+6 q+4, g^{*}\left(u_{q+2}\right) \equiv 2 p q+6 q+12, g^{*}\left(u_{q+3}\right) \equiv 2 p q+6 q+$ $14, g^{*}\left(u_{q+4}\right) \equiv 2 p q+6 q+16, g^{*}\left(u_{q+5}\right) \equiv 2 p q+6 q+22, g^{*}\left(u_{q+6}\right) \equiv 2 p q+$ $6 q+28, g^{*}\left(u_{q+7}\right) \equiv 2 p q+6 q+30, g^{*}\left(u_{q+8}\right) \equiv 2 p q+6 q+32, g^{*}\left(u_{q+9}\right) \equiv$ $2 p q+6 q+38, g^{*}\left(u_{q+10}\right) \equiv 2 p q+6 q+44, \cdots, g^{*}\left(u_{2 q-8}\right) \equiv 2 p q+10 q-$ $30, g^{*}\left(u_{2 q-7}\right) \equiv 2 p q+10 q-28, g^{*}\left(u_{2 q-6}\right) \equiv 2 p q+10 q-22, g^{*}\left(u_{2 q-5}\right) \equiv$ $2 p q+10 q-16, g^{*}\left(u_{2 q-4}\right) \equiv 2 p q+10 q-14, g^{*}\left(u_{2 q-3}\right) \equiv 2 p q+10 q-$ $12, g^{*}\left(u_{2 q-2}\right) \equiv 2 p q+10 q-6, g^{*}\left(u_{2 q-1}\right) \equiv 2 p q+10 q, g^{*}\left(u_{2 q}\right) \equiv 2 p q+$ $10 q+2$.

The labels of the vertices of circles $C_{q}^{(3)}, C_{q}^{(4)}, \cdots, C_{q}^{(p)} \bmod 4 p q$ as the same in case (1).

Illustration: The e. e. g. l. of $T_{9,9}, T_{11,9}, T_{13,9}$ and $T_{15,9}$ are presented in Figure 15, respectively.

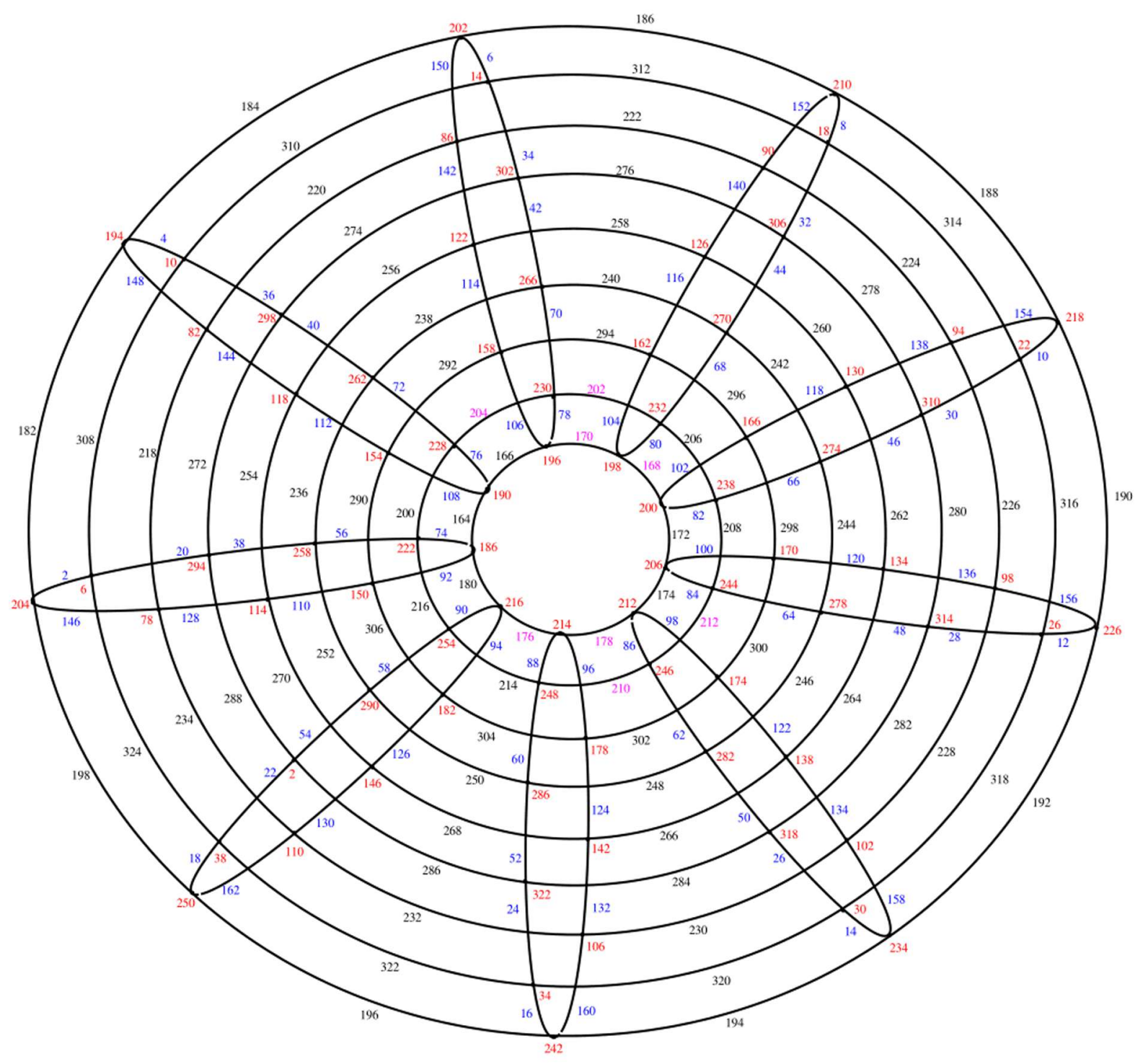

(a) $T_{9,9}$ 


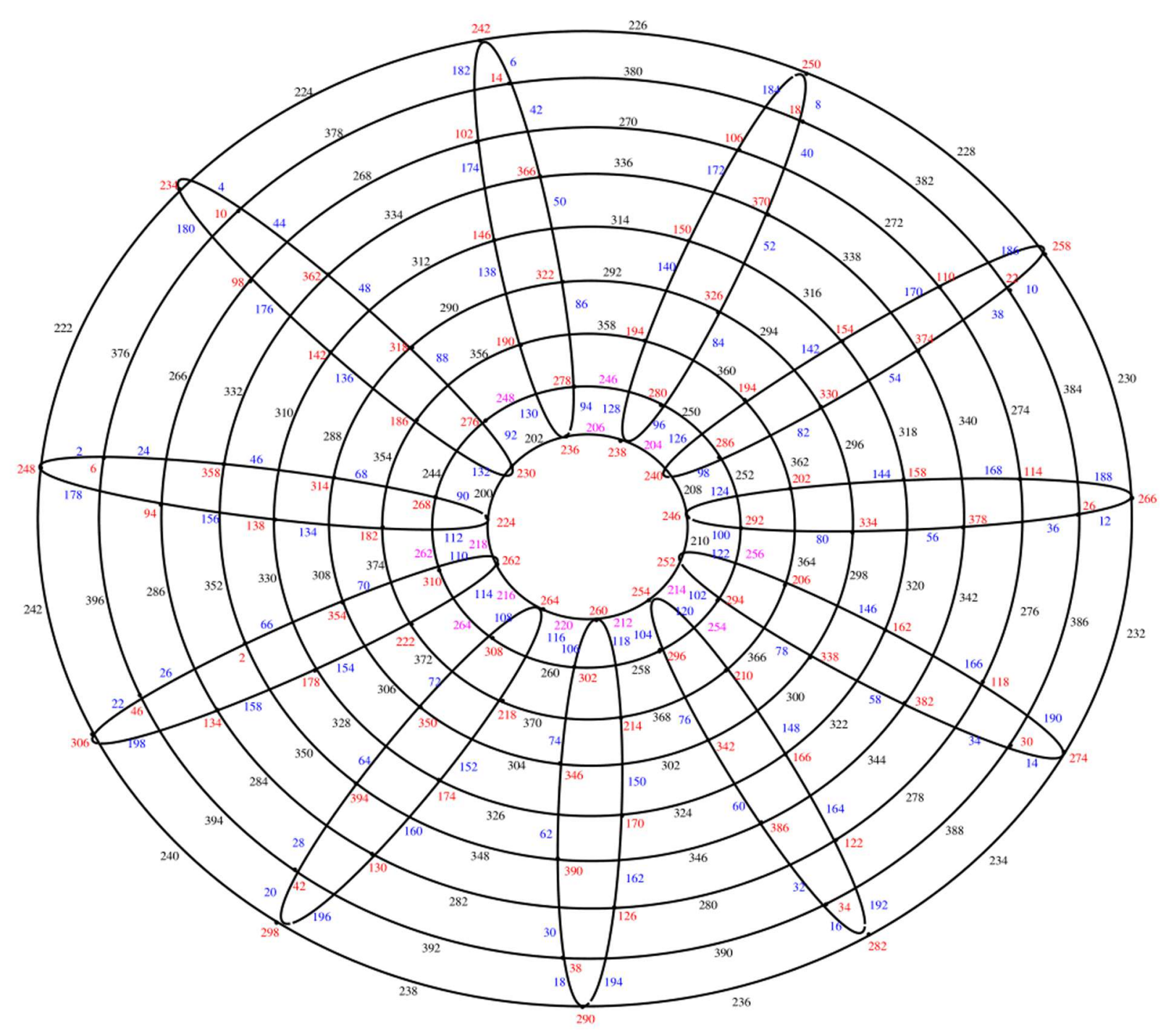

(b) $T_{11,9}$ 


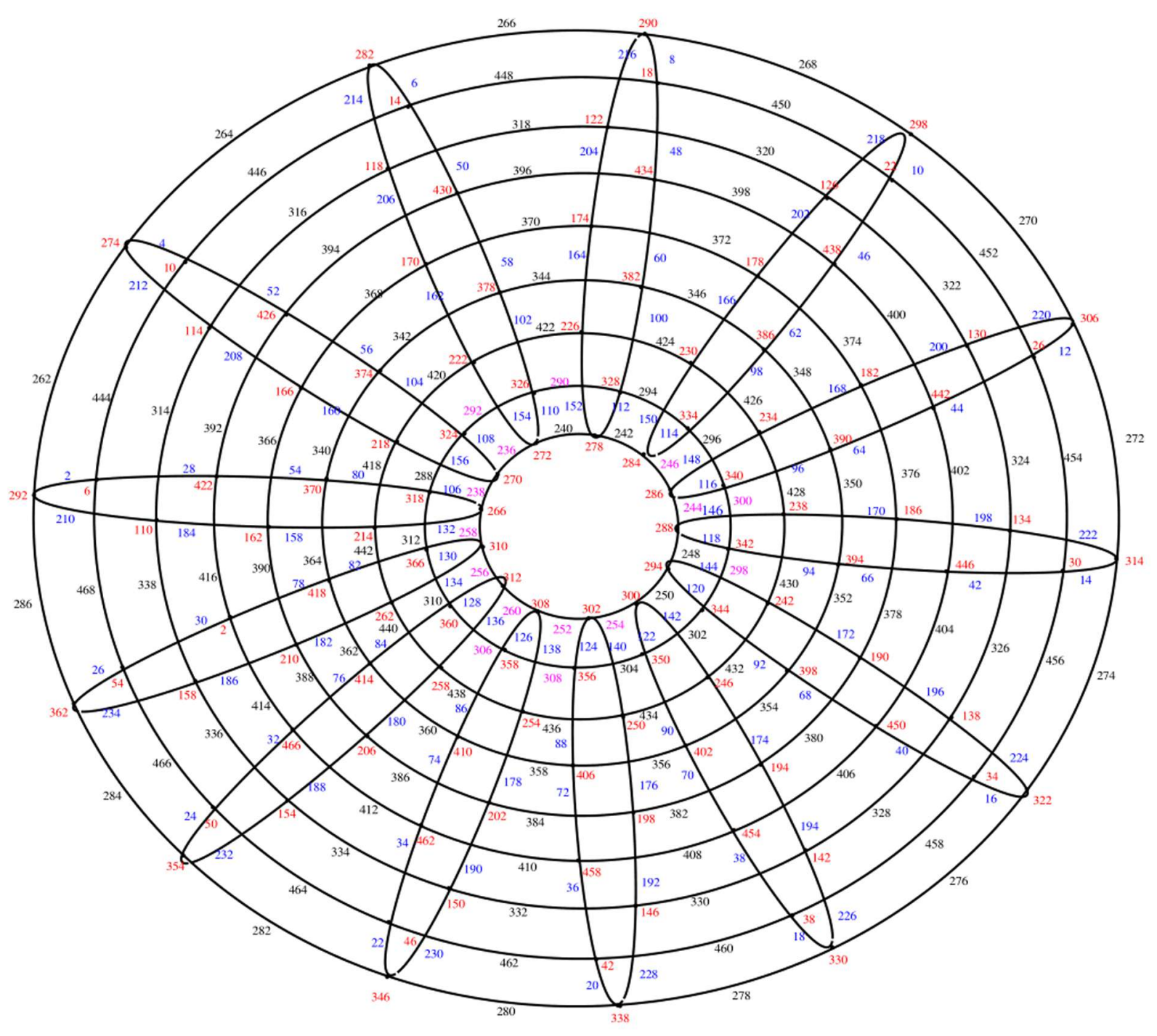

(c) $T_{13,9}$ 


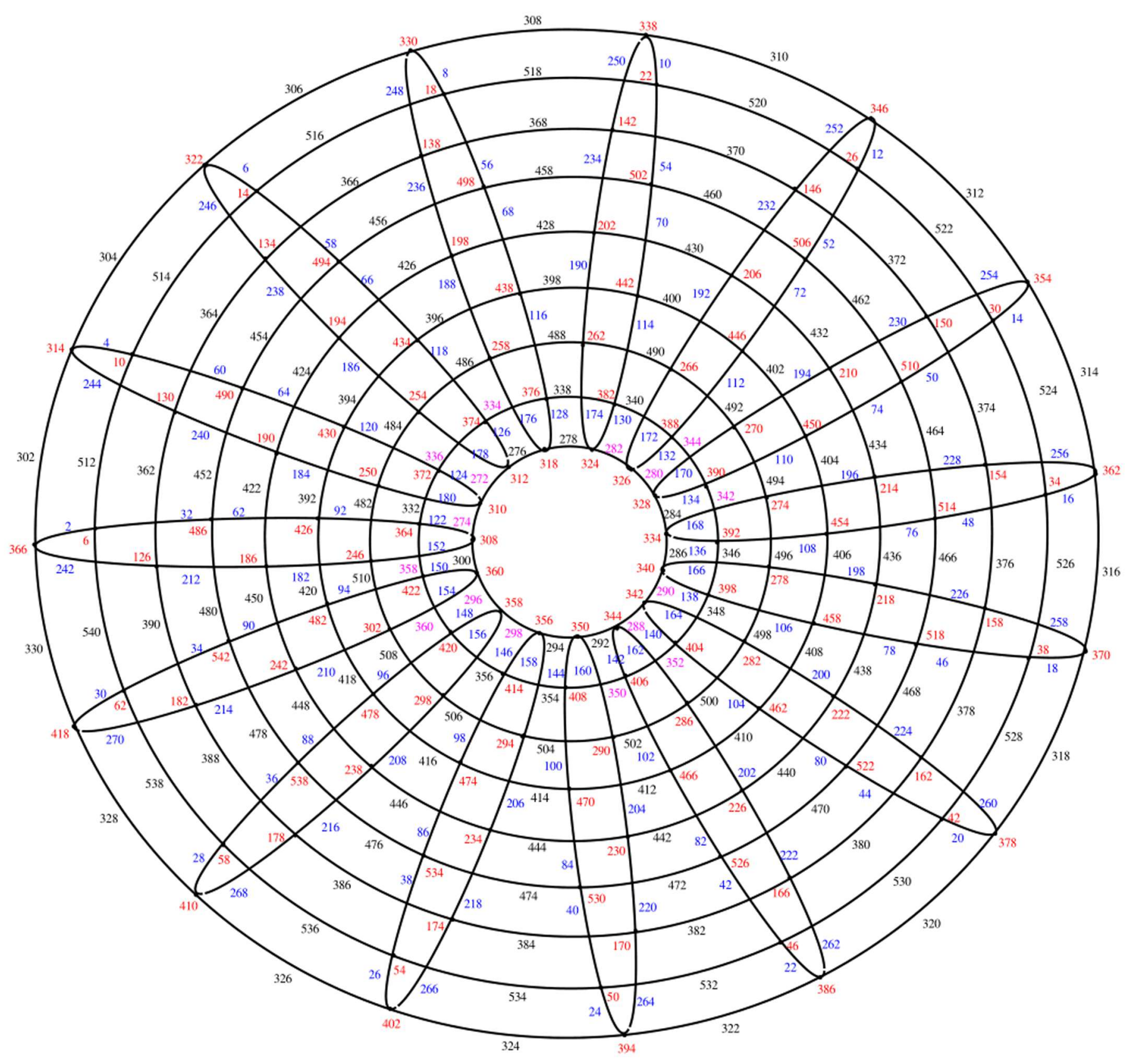

(d) $T_{15,9}$

Figure 2.15: The e. e. g. 1. of $T_{9,9}, T_{11,9}, T_{13,9}$ and $T_{15,9}$ 


\section{Conclusion}

In this paper, we have used the connection of labeling of graphs with modular arithmatic and the theory of numbers in general. Also, we have given a detailed study for e.e.g.l. of all cases of members of the torus grid graphs. Moreover, we have given the necessary and sufficient conditions for e.e.g.l. of other important families which is including rectangular $P_{m} \times P_{n}$ grid graphs that should be taken into consideration in future studies of e.e.g.l.

\section{References}

[1] B. D. Acharya, S. Arumugam, and A. Rosa, Eds., Labelings of discrete structures and applications. New Delhi: Narosa, 2008.

[2] M. Alaeiyan and M. R. Farahani, "The 1-2-3-edge labeling and vertex colors", International journal of applied mathematics and machine learning, vol. 4, no. 2, Jun. 2016, pp. 119-133, doi: 10.18642/ijamml_7100121608

[3] G. S. Bloom, "Numbered undirected graphs and their uses. A survey of a unifying scientific and engineering concept and its use in developing a theory of non-redundant homometric sets relating to some ambiguities in X-ray Diffraction analysis", Ph. D. Thesis, University of Southern California, Los Angeles, 1975. [On line]. Available: https://bit.ly/3fiWqwd

[4] G. S. Bloom and S. W. Golomb, "Applications of numbered undirected graphs", Proceedings of the IEEE, vol. 65, no. 4, pp. 562-570, Apr. 1977, doi: $10.1109 /$ proc.1977.10517

[5] G. S. Bloom and S. W. Golomb, "Numbered complete graphs, unusual rulers, and assorted applications", in Theory and applications of graphs, Y. Alavi and D. R. Lick, Eds. Berlin: Springer, 1978, pp. 53-65, doi: $10.1007 / \mathrm{bfb} 0070364$

[6] G. S. Bloom and D. F. Hsu, "On graceful digraphs and a problem in network addressing", Congressus numerantium, vol. 35, pp. 91-103, 1982. 
[7] S. N. Daoud, "Edge odd graceful labeling of some path and cycle related graphs", AKCE international journal of graphs and combinatorics, vol. 14, no. 2, pp. 178-203, 2017, doi: 10.1016/j.akcej.2017.03.001

[8] S. N. Daoud, "Edge odd graceful labeling Cylinder grid and Torus grid graphs", IEEE access , vol. 7, pp. 10568-10592, 2018, doi:10.1109/access.2018.2889293

[9] S. N. Daoud, "Edge even graceful labeling polar grid graph", Symmetry, vol. 11, no. 1, Art ID. 38, Jan. 2019, doi:10.3390/sym11010038

[10] S. N. Daoud, "Vertex odd graceful labeling", Ars combinatoria, vol. 142 , pp. 65-87, 2019. [On line]. Available: https://bit.ly/20b4gMN

[11] A. Elsonbaty and S. N. Daoud, "Edge even graceful labeling of some path and cycle related graphs", Ars combinatoria, vol. 130, no. 2, pp. 79-96, 2017.

[12] M. R. Farahani, "On the 1-2-3-edge weighting and vertex coloring of complete graph", International journal of computational sciences \& applications, vol. 3, no. 3, Jun. 2013, pp. 19-23, doi: 10.5121/ijcsa.2013.3302

[13] M. R. Farahani and S. H. Hosseini, "The 1-2-3-edge labeling and vertex coloring of complete graph Kn with a modified algorithm", Algebras, groups and geometries, vol. 31, no. 2, 2014, pp. 183-199. [On line]. Available: https://bit.ly/2ZmbuUF

[14] J. A. Gallian, "Graph labeling", The electronic journal of combinatorics, vol. DS6, Dec. 2017, doi: 10.37236/27

[15] Z. Gao, "Odd graceful labelings of some union graphs", Journal of natural science of Heilongjiang University, no. 1, pp. 35 -39, 2007. [On line]. Available: https://bit.ly/2CliUOT

[16] R. B. Gnanajothi, "Topics in graph theory", Ph. D. Thesis, Madurai Kamaraj University, 1991.

[17] S. W. Golomb, "How to number a graph", in Graph theory and computing, R. C. Read, Ed., New York, NY: Academic Press, 1972, pp. 23-37, doi: 10.1016/b978-1-4832-3187-7.50008-8

[18] R. L. Graham and H. O. Pollak, "On the addressing problem for loop switching", The Bell system technical journal, vol. 50, no. 8, 1971, pp. 2495-2519, doi: 10.1002/j.1538-7305.1971.tb02618.x 
[19] J. Gross and J. Yellen, Graph theory and its applications, Philadelphia, PA: CRC Press, 1999.

[20] Q. Kuan, S. Lee, J. Mitchem, and A. Wang, “On edge-graceful unicyclic graphs", Congressus numerantium, vol. 61, pp. 65-74, 1988.

[21] L. Lee, S. Lee, and G. Murty, "On edge-graceful labelings of complete graphs solution of Lo's conjecture", Congressus numerantium, vol. 62, pp. 225-233, 1988.

[22] Li Yan, Yingfang Li, Xiujun Zhang, Madiha Saqlain, Sohail Zafar, and. M. R. Farahani, "Total edge product cordial labeling of some new classes of graphs", Journal of information \& optimization sciences, vol. 39, no. 3, pp. 705-724, 2018, doi: 10.1080/02522667.2017.1417727

[23] S. P. Lo, "On edge-graceful labelings of graphs", Congressus numerantium, vol. 50, pp. 231-241, 1985.

[24] A. Rosa, "On certain valuations of the vertices of a graph," in Theory of Graphs, International Symposium, Rome, July 1966, New York, NY: Gordon and Breach, 1967, pp. 349-355.

[25] M. A. Seoud and M. E. Abdel-Aal, "On odd graceful graphs", Ars combinatoria, vol. 108, pp. 161-185, 2013.

[26] Y. Shang, "Geometric assortative growth model for small-world networks", The scientific world journal, vol. 2014, Art ID. 59391, Jan. 2014, doi:10.1155/2014/759391

[27] Y. Shang, "More on the normalized Laplacian Estrada index", Applicable analysis and discrete mathematics, vol. 8, no. 2, pp. 346-357, 2014, doi: 10.2298/aadm140724011s

[28] A. Solairaju and K. Chithra, "Edge-odd graceful graphs", Electronic notes in discrete mathematics, vol. 33, pp. 15-20, Apr. 2009, doi: 10.1016/j.endm.2009.03.003

[29] M. Sutton, "Summable graphs labellings and their applications", Ph. D. Thesis, The University of Newcastle, Callaghan, 2001.

[30] H. Yang, M. A. Rashid, S. Ahmad, M. K. Siddiqui, and M. F. Hanif, "Cycle super magic labeling of pumpkin, octagonal and hexagonal graphs", Journal of discrete mathematical sciences and cryptography, vol. 22, no. 7, pp. 1165-1176, 2019, doi: 10.1080/09720529.2019.1698800 